\title{
Refining G-structure classifications
}

\author{
Oisín A. P. Mac Conamhna* \\ DAMTP \\ Centre for Mathematical Sciences \\ University of Cambridge \\ Wilberforce Road, Cambridge CB3 0WA, UK.
}

\begin{abstract}
Using G-structure language, a systematic, iterative formalism for computing neccessary and sufficient conditions for the existence of $N$ arbitrary linearly independent Killing spinors is presented. The key organisational tool is the common isotropy group of the Killing spinors. The formalism is illustrated for configurations in gauged $S U(2)$ supergravity in seven dimensions admitting at least one null Killing spinor, and the possible isotropy groups are shown to be $\left(S U(2) \ltimes \mathbb{R}^{4}\right) \times \mathbb{R}, S U(2), \mathbb{R}^{5}$, or the identity. The constraints associated with the existence of certain additional Killing spinors are computed, and used to derive numerous solutions. A discussion of the relevance of the formalism to the complete classification of all supersymmetric configurations in $d=11$ is given.
\end{abstract}

KEYWORDS: G-structures, supergravity.

*O.A.P.MacConamhna@damtp.cam.ac.uk 


\section{Contents}

1. Introduction 2

2. The supergravity 10

3. Configurations admitting at least one null Killing spinor 11

3.1 The G-structure [1]

3.2 Constraints for supersymmetry 13

3.2.1 Constraints from $\delta \lambda=0$

$3.2 .2 \delta \psi_{\mu}=0$

3.3 Introducing coordinates 16

3.4 Refining the classification 17

田. $\left(S U(2) \ltimes \mathbb{R}^{4}\right) \times \mathbb{R}$ structure 18

4.1 Constraints 18

4.2 Examples 20

5. $S U(2)$ structure 20

5.1 One additional Killing spinor 20

$5.2 S U(2)$ structures with eight supersymmetries 23

5.3 Examples 26

$5.3 .1 \quad A d S_{3} \times \mathcal{M}_{4}$ solutions 26

5.3.2 Generalisations: membranes with $A d S_{3}$ worldvolume 29

6. $\mathbb{R}^{5}$ structure 32

7. Identity Structure 33

7.1 Constraints 34

7.2 Examples 35

8. Conclusions and outlook 38

9. Acknowledgements 39

目. Conventions 39

B. Useful projections 41

G. Spin connection components 41 


\section{Introduction}

In recent years, it has been realised that the notion of a G-structure is a powerful tool in classifying supersymmetric geometries in supergravity theories. It has been succesfully and fruitfully applied in numerous interesting contexts, for example [1][19]. The strategy is simple. One assumes the existence of at least one Killing spinor. The existence of a Killing spinor is equivalent to the existence of a set of globally defined bilinears, specifying the G-structure, which are invariant under the isotropy group $G$ of the spinor. Applying the Fierz identities to the bilinears allows the deduction of algebraic relations between them. Next applying the Killing spinor equation to the bilinears determines the intrinsic torsion of the G-structure in terms of the bosonic fields of the theory. If the theory contains any additional fermions, the vanishing of their supersymmetry variations implies additional algebraic relationships between the bosonic fields. When one has derived the complete set of constraints, one substitutes them back into the supersymmetry transformations and shows that they are also sufficient for supersymmetry. One thus arrives at a set of necessary and sufficient conditions for a bosonic configuration to admit at least one Killing spinor. However the existence of at least one Killing spinor generically implies that some but not all of the equations of motion and Bianchi identities are identically satisfied, so some subset thereof must still be imposed on the most general field configuration consistent with the constraints. The standard G-structure scheme should thus be thought of as providing the most general supersymmetric ansatz for the supergravity in question.

The standard G-structures formalism has proven to be extremely powerful, and useful from the point of view of explicitly constructing new solutions, in simple lower dimensional supergravities with few supercharges. In these simple cases the computational effort required to arrive at the general ansatz is also fairly minimal. However as the dimensionality of spacetime and/ or the number of supercharges increases, the constraints implied by the existence of a single Killing spinor become proportionately weaker, and the most general ansatz becomes broader. Though the reduced problem of solving the remaining field equations for the most general supersymmetric ansatz is very much simpler than trying to solve the original full set ab initio, it is generically hard to do. For example, while it is conceptually beautiful that the most general supersymmetric ansatz for $d=11$ supergravity may be explicitly computed [9], [10], the ansatz is not enormously useful, given current techniques, when it comes to explicitly generating new solutions. Furthermore, calculating the ansatz 
for more complicated theories using the standard approach requires a lot of (in fact, largely redundant) computation. For a theory such as IIB, the ammount of calculation required to derive the ansatz using the standard procedure would be truly gargantuan.

There are thus two related improvements to the standard procedure which are required to fully realise the power the G-structure formalism. The first, of a purely technical nature, is to give a more efficient means of performing the calculations. The second is to give a systematic formalism for classifying configurations preserving more than one supersymmetry. Some attempts in this direction have been made, for simple supergravities, using a mixture of G-structure and integrability techniques [2], [18], [19]. However this approach will not really be viable for more complicated theories; it would be preferable to have a universally applicable formalism which uses G-structure language throughout, as was done (in a purely Riemannian context) in [7]. Requiring a configuration to admit more than one Killing spinor would imply more constraints on the bosonic fields of the theory. Furthermore it would also imply that more of the field equations and Bianchi identities would be identically satisfied. Thus a refined G-structure classification scheme would be much more useful from the point of view of explicitly constructing supersymmetric solutions. The objective of this paper is to propose a means of implementing these improvements to the standard procedure, illustrated in the context of $S U(2)$ gauged supergravity in seven dimensions, a theory with sixteen real supercharges.

The technical novelty employed, which in fact renders a refined classification tractable, is to fully exploit throughout the calculation a point raised in [10]. The point is that any spinor defines a preferred orthonormal basis of spacetime, and in this basis the associated G-structure simplifies dramatically, and the spinor is in fact constant. For example, in the seven dimensional context of this paper, we will see below that a single null spinor defines an $\left(S U(2) \ltimes \mathbb{R}^{4}\right) \times \mathbb{R}$ structure. A single pair of null symplectic majorana spinors (we will work with symplectic majorana spinors throughout) may be fixed by the projections

$$
\begin{aligned}
\Gamma^{12} \epsilon^{1} & =i \epsilon^{2}, \\
\Gamma^{13} \epsilon^{1} & =-\epsilon^{2}, \\
\Gamma^{14} \epsilon^{1} & =i \epsilon^{1}, \\
\Gamma^{5} \epsilon^{a} & =\epsilon^{a},
\end{aligned}
$$

in the basis

$$
d s^{2}=-2 e^{+} e^{-}+\delta_{i j} e^{i} e^{j}+\left(e^{5}\right)^{2} .
$$

The four dimensional Riemannian manifold with metric $\delta_{i j} e^{i} e^{j}$ will be referred to as the base. The various bilinears may be trivially computed without having to invoke the full Fierzing machinery, and have constant components in this spacetime basis. This is what was done in [10]. 
We will take this simplification a stage further. The supergravity we analyse has two fermions, $\lambda$ and $\psi_{\mu}$. Consider first the constraints implied by the vanishing of $\delta \lambda$. The standard way of analysing these would be to contract $\delta \lambda=0$ with all spinors of the form $\bar{\epsilon} \Gamma^{(n)}$ up to three gamma matrices to form all possible combinations of bilinears, and deduce the implied constraints on the bosonic fields. However it is much more efficient to first impose the projections (1.1). Then as we shall see below, $\delta \lambda=0$ may be cast in the schematic form

$$
\delta \lambda=\left(q+i q^{A} T^{A}+q^{i} \Gamma_{i}+\left(r+i r^{A} T^{A}\right) \Gamma^{-}+r^{i} \Gamma^{-} \Gamma_{i}\right) \epsilon=0,
$$

where the $T^{A}$ are the Pauli matrices, and we have suppressed symplectic Majorana indices. In doing this, we shall see below that we are rewriting $\delta \lambda$ manifestly in terms of a basis for spinor space; by linear independence, each of the $q, q^{A}, q^{i}, r$, $r^{A}, r^{i}$ must vanish separately. By imposing the projections and decomposing in this fashion, it is very much quicker to derive the constraints. Next consider the Killing spinor equation. The standard procedure for analysing the differential constraints would be to apply the full Killing spinor equation to each of the bilinears to deduce the various components of the spin connection. Again, this is not efficient. In its preferred basis, the spinor $\epsilon$ has constant components, so in this basis the Killing spinor equation becomes schematically

$$
\delta \psi_{\mu}=\frac{1}{4} \omega_{\mu \nu \sigma} \Gamma^{\nu \sigma} \epsilon+\text { fluxes }=0
$$

and thus yields algebraic relations between the fluxes and the spin connection in the preferred basis. Again we may impose the various defining projections on $\epsilon$ to reduce each spacetime component of the Killing spinor equation to the schematic form

$$
\delta \psi_{\mu}=\left(q_{\mu}+i q_{\mu}^{A} T^{A}+q_{\mu}^{i} \Gamma_{i}+\left(r_{\mu}+i r_{\mu}^{A} T^{A}\right) \Gamma^{-}+r_{\mu}^{i} \Gamma^{-} \Gamma_{i}\right) \epsilon=0
$$

Requiring by linear independence that each term in each spacetime component of the Killing spinor equation vanishes separately allows one to fix the spin connection in terms of the fluxes, and thus deduce the most general supersymmetric ansatz with a minimum of effort. This streamlined way of computing the constraints has also recently been advocated in 20.

Having sketched the technical innovation used to reduce as much as possible the ammount of computation required for the broadest ansatz, we now turn to the question of refining the classification. We will assume the existence of the single null Killing spinor defined by the projections (1.1), and wish to compute the further constraints on the bosonic fields of the theory for it to admit an arbitrary additional linearly independent Killing spinor. An important observation in organising the refined classification is the following. Incorporating additional Killing spinors can have one of two effects. Either the existence of an additional Killing spinor implies a 
further global reduction of the structure group of the frame bundle (so the structure group is reduced from $G$ to some subgroup), or it implies additional restrictions on the intrinsic torsion of the existing G-structure. To illustrate this point, consider the basis of sixteen spinors defined by the projections (with no sum on $i$ )

$$
\begin{aligned}
\Gamma^{12} \epsilon_{(i)}^{1} & =i \alpha_{(i)}^{1} \epsilon_{(i)}^{2}, \\
\Gamma^{13} \epsilon_{(i)}^{1} & =-\alpha_{(i)}^{2} \epsilon_{(i)}^{2}, \\
\Gamma^{14} \epsilon_{(i)}^{1} & =i \alpha_{(i)}^{3} \epsilon_{(i)}^{1}, \\
\Gamma^{5} \epsilon_{(i)}^{a} & =\alpha_{(i)}^{4} \epsilon_{(i)}^{a},
\end{aligned}
$$

for all sixteen possible combinations of $\alpha^{1}, \ldots, \alpha^{4}= \pm 1$. All these spinors are null, and they are all constant in the spacetime basis (1.2). We denote them by $\left(\alpha_{1}, \alpha_{2}, \alpha_{3}, \alpha_{4}\right)$, and also introduce the notation

$$
\begin{aligned}
& \prod_{j=1}^{4} \alpha^{j}=\gamma \\
& \prod_{A=1}^{3} \alpha^{A}=\beta
\end{aligned}
$$

Our basis spans the space of spinors in the theory, so any additional Killing spinor, whether timelike or null, must be a linear combination of these spinors; for an arbitrary additional Killing spinor $\epsilon_{K}$,

$$
\epsilon_{K}=\sum_{i=1}^{N} f_{(i)} \epsilon_{(i)}
$$

where the sixteen $f_{(i)}$ are real functions. We may rewrite our basis spinors in terms of our fiducial Killing spinor $\epsilon=(+,+,+,+$,$) ; the three other basis spinors with$ $\gamma=1, \beta=1$ may be written as

$$
i T^{A} \epsilon
$$

since these obey the appropriate projections. Next the four basis spinors with $(\gamma, \beta)=(+,-)$ are given by

$$
\Gamma^{i} \epsilon
$$

while the two sets of four basis spinors with $(\gamma, \beta)=(-,+),(-,-)$ are

$$
\left(\Gamma^{-} \epsilon, i \Gamma^{-} T^{A} \epsilon\right) ; \quad \Gamma^{-} \Gamma^{i} \epsilon,
$$

respectively. Hence an arbitrary additional linearly independent Killing spinor is given by

$$
\epsilon_{K}=\left(\delta+i \delta^{A} T^{A}+\delta_{i} \Gamma^{i}+\Gamma^{-}\left(\theta+i \theta^{A} T^{A}+\theta_{i} \Gamma^{i}\right)\right) \epsilon
$$


and we recognise in equations (1.3) and (1.5) precisely the decomposition of the supersymmetry variations on the basis. Now, we will compute the common isotropy group of the spinor $\epsilon_{K}$ and the fiducial Killing spinor $\epsilon$ for various choices of the sixteen functions specifying $\epsilon_{K}$. We note that basis spinors with $\gamma=1$ are annihilated by $\Gamma^{+}$:

$$
\Gamma^{+} \epsilon_{(i)}=0, \quad \gamma_{(i)}=1,
$$

while basis spinors with $\gamma=-1$ are annihilated by $\Gamma^{-}$,

$$
\Gamma^{-} \epsilon_{(i)}=0, \quad \gamma_{(i)}=-1
$$

Furthermore, since $\beta$ labels minus the chirality of the basis spinors on the four dimensional base, anti selfdual linear combinations of the $\Gamma^{i j}$ annihilate basis spinors with $\beta=1$; for a two form $A_{i j}^{(-)}$which is anti selfdual on the base but otherwise arbitrary,

$$
A_{i j}^{(-)} \Gamma^{i j} \epsilon_{(i)}=0, \quad \beta_{(i)}=1 .
$$

Throughout this work, for any object with two antisymmetric indices $i, j$ on the base, the superscripts $(+)$ and $(-)$ will denote respectively the selfdual and antiselfdual projections on $i, j$. Our choice of orientation, together with all other conventions, is given in Appendix A. Next, basis spinors with $\beta=-1$ are annihilated by selfdual linear combinations; for an arbitrary selfdual form $A_{i j}^{(+)}$,

$$
A_{i j}^{(+)} \Gamma^{i j} \epsilon_{(i)}=0, \quad \beta_{(i)}=-1 .
$$

Therefore, the most general element of the Lie algebra of $\operatorname{Spin}(1,6)$ which annihilates the fiducial Killing spinor $\epsilon$ is

$$
B^{A} K_{i j}^{(-) A} \Gamma^{i j}+B_{i} \Gamma^{+i}+B \Gamma^{+5}
$$

which generates the group $\left(S U(2) \ltimes \mathbb{R}^{4}\right) \times \mathbb{R}$; the $K^{A}$ are a triplet of anti selfdual forms obeying the quaternionic algebra. However, the three additional Killing spinors with $(\gamma, \beta)=(+,+)$ are also annihilated precisely by (1.18); all the spinors in the four dimensional subspace spanned by the $(+,+)$ basis spinors share the same isotropy group. Thus, additional linearly independent Killing spinors of the form

$$
\epsilon_{K}=\left(\delta+i \delta^{A} T^{A}\right) \epsilon
$$

do not imply any further reduction of the structure group. However, they will imply further restrictions on the intrinsic torsion of the $\left(S U(2) \ltimes \mathbb{R}^{4}\right) \times \mathbb{R}$ structure. Since none of the other twelve basis spinors are annihilated by (1.18), we see that an $\left(S U(2) \ltimes \mathbb{R}^{4}\right) \times \mathbb{R}$ structure is compatible with having one, two, three or four linearly independent Killing spinors. 
Next consider Killing spinors which are linear combinations of $(+,+)$ and $(-,+)$ spinors,

$$
\epsilon_{K}=\left(\delta+i \delta^{A} T^{A}+\Gamma^{-}\left(\theta+i \theta^{A} T^{A}\right)\right) \epsilon,
$$

with at least one of the $\theta, \theta^{A} \neq 0$. An arbitrary $(-,+)$ spinor is annihilated by a different $\left(S U(2) \ltimes \mathbb{R}^{4}\right) \times \mathbb{R} \subset \operatorname{Spin}(1,6)$; the most general Lie algebra element annihilating such a spinor is

$$
B^{A} K_{i j}^{(-) A} \Gamma^{i j}+B_{i} \Gamma^{-i}+B \Gamma^{-5} .
$$

Thus in this case the common isotropy group of the spinors $\epsilon, \epsilon_{K}$ is $S U(2)$, generated by

$$
B^{A} K_{i j}^{(-) A} \Gamma^{i j},
$$

and so additional Killing spinors of the form (1.20) reduce the structure sroup to $S U(2)$. There are eight linearly independent spinors with this common isotropy group, so given the existence of the fiducial Killing spinor, an $S U(2)$ structure is compatible with the existence of $2,3, \ldots, 8$ linearly independent Killing spinors. The more Killing spinors there are, the more restrictive the constraints on the torsion will be.

It is clear how to proceed. Next consider a Killing spinor of the form

$$
\epsilon_{K}=\left(\delta+i \delta^{A} T^{A}+\delta_{i} \Gamma^{i}\right) \epsilon,
$$

with at least one of the $\delta_{i} \neq 0$. The $\left(S U(2) \ltimes \mathbb{R}^{4}\right) \times \mathbb{R}$ isotropy group of a $(+,-)$ spinor is generated by

$$
B^{A} J_{i j}^{(+) A} \Gamma^{i j}+B_{i} \Gamma^{+i}+B \Gamma^{+5},
$$

so in this case the common isotropy group of the spinors $\epsilon, \epsilon_{K}$ is $\mathbb{R}^{5}$, generated by

$$
B_{i} \Gamma^{+i}+B \Gamma^{+5} .
$$

As in the $S U(2)$ case, there are eight linearly independent spinors with this common isotropy group, so given the existence of the fiducial Killing spinor, an $\mathbb{R}^{5}$ structure is compatible with the existence of $2, \ldots, 8$ linearly independent Killing spinors.

It is easy to verify that assuming the existence of Killing spinors which are more general linear combinations of the basis spinors than the three cases discussed above imply that the structure group is reduced to the identity. Such a structure, given the existence of the fiducial Killing spinor, is compatible with the existence of $2,3, \ldots, 16$ linearly independent Killing spinors.

In the refinement of the G-structure classification scheme proposed here, configurations admitting multiple linearly independent Killing spinors are naturally classified according to the structure group, rather than the number of Killing spinors. We have seen how we can have $G=\left(S U(2) \ltimes \mathbb{R}^{4}\right) \times \mathbb{R}$ and four linearly independent Killing 
spinors, or $G=I d$ and only two. This is somewhat different to other approaches, for example generalised holonomy [21]-23], where the aim has always been to classify configurations according to the number of preserved supersymmetries. From our perspective, this is rather unnatural; the most significant feature of a supersymmetric configuration in the G-structure formalism is the structure group. Then for a given structure group, demanding more supersymmetries imposes progressively more severe constraints on the intrinsic torsion.

The most efficient way that we have been able to find of computing the additional constraints implying and implied by the existence of arbitrary additional Killing spinors is the following. Denote the supersymmetry variations $\delta \lambda, \delta \psi$ with parameter $\epsilon_{K}$ as $\Delta_{\lambda} \epsilon_{K}, \mathcal{D}_{\mu} \epsilon_{K}$. Since we may write $\epsilon_{K}=Q \epsilon$, for some $Q$ of the form of equation (1.13), we observe that since $\epsilon$ is Killing, $\epsilon_{K}$ is Killing if and only if

$$
\begin{aligned}
& {\left[\Delta_{\lambda}, Q\right] \epsilon=0} \\
& {\left[\mathcal{D}_{\mu}, Q\right] \epsilon=0 .}
\end{aligned}
$$

Having evaluated the commutators, we may as before impose the projections satisfied by $\epsilon$ to reduce these expressions to manifest linear combinations of the basis spinors, and by linear independence, each coefficient must vanish separately. The procedure may be iterated at will, for any desired choices of $Q$, consistent with any desired structure group. For simple supergravities, such as those with eight supercharges, the procedure given here should be easy to employ to perform a fully refined classification, and should involve a modest amount of calculation. For a theory of the complexity of the one studied in this paper, with sixteen supercharges, the amount of computation required to perform a complete classification is much larger. This is because the most general additional linearly independent Killing spinor consistent with the existence of an identity structure is parameterised by sixteen real functions, and keeping track of all the terms in (1.26), (1.27) is technically involved. Additional Killing spinors consistent with the larger structure groups $\left(S U(2) \ltimes \mathbb{R}^{4}\right) \times \mathbb{R}, S U(2)$ and $\mathbb{R}^{5}$ are somewhat easier to handle, since these are parameterised by four, eight and eight real functions respectively. Nevertheless, we have not performed the refined classification in its entirety; for the smaller structure groups, we have restricted to some illustrative examples, rather than pursuing the problem in full generality. However, we emphasise that there is no conceptual difficulty in doing so; the calculations involved, while lengthy, are simple and repetitive, involving nothing more than evaluating gamma-matrix commutators and imposing a fixed set of spinor projections.

The traditional approach to finding supersymmetric solutions of supergravity theories has been to make some ansatz for the bosonic fields, and then to use the supersymmetry variations of the fermions to determine if there are any Killing spinors consistent with that ansatz. The G-structures programme can be thought of as the 
exact converse of this; one makes an ansatz for the Killing spinors, and expresses the conditions for a spinorial solution of the supersymmetry variations of that form to exist as a set of constraints on the bosonic fields. The power of the formalism lies in the fact that the spinor ansatz can, if desired, be made completely general, whereby we mean that the constraints on the bosonic fields for $N$ arbitrary linearly independent Killing spinors (for any desired structure group) to exist can be evaluated, albeit with some effort, in any theory. What we have argued above is that the formidably complicated problem of determining the constraints on the bosonic fields for the general multi-spinor solution of the fermion supersymmetry transformations to exist may naturally be organised into more manageable subproblems, with G-structures providing the organising principle. One may classify the possible types of multi-spinor ansätze according to the common isotropy group of the spinors. Then within each class one may deduce the constraints on the intrinsic torsion of the structure implied by the existence of any desired number of arbitrary linearly independent spinors within that class.

The remainder of this paper is organised as follows. In section two we briefly describe $S U(2)$ gauged supergravity in seven dimensions. In section three we perform a streamlined analysis of the constraints implying and implied by the existence of a single null Killing spinor, and introduce coordinates for the problem. In section four, with a modest assumption of the form of the Yang-Mills fields of the theory introduced for computational convenience, we perform a complete classification of multi-supersymmetric configurations admitting a strictly $\left(S U(2) \ltimes \mathbb{R}^{4}\right) \times \mathbb{R}$ structure. It is shown that a second Killing spinor with the same structure group as the first exists if and only if the Yang-Mills fields are truncated to a $U(1)$ subgroup. The existence of a third Killing spinor with the same isotropy group implies and is implied by the vanishing of the Yang-Mills fields, and implies the existence of a fourth Killing spinor. In section five we study configurations with an $S U(2)$ structure; neccessary and sufficient conditions for the existence of an additional Killing spinor of a specific form are derived, and also all configurations admitting eight Killing spinors fixed by the same $S U(2)$ are explicitly classified. We illustrate the effect incorporating additional Killing spinors has on the torsion of the $S U(2)$ structure by deriving known $A d S_{3}$ solutions of the theory from our ansatz, and generalise these solutions to construct (singular) membrane solutions with $A d S_{3}$ worldvolumes. Configurations with $\mathbb{R}^{5}$ structures are the topic of section six. In section seven we discuss identity structures, providing explicit examples. Section seven contains our conclusions, and a discussion of the applicability of the formalism to the complete classification of supersymmetric configurations in eleven dimensions. In appendix A we give our conventions, and appendix B lists a set of projections satisfied by $\epsilon$ which we use throughout. In appendix $\mathrm{C}$ we give the components of the spin connection. Appendix D contains the integrability conditions for the theory. 


\section{The supergravity}

Minimal $S U(2)$ gauged supergravity in seven dimensions was first constructed in [24] but with numerical typos, which were corrected in [25]. In our conventions, the bosonic lagrangian density for the theory is

$$
\begin{aligned}
e^{-1} \mathcal{L} & =\frac{1}{2} R-\frac{1}{24}\left(G_{\mu \nu \rho \tau}\right)^{2}-\frac{1}{2} F_{\mu \nu}{ }_{b}{ }_{b} F^{\mu \nu b}{ }_{a}-\frac{5}{2}\left(\partial_{\mu} \phi\right)^{2}+\frac{h}{18} e^{-2 \phi} \epsilon^{\mu \nu \sigma \tau \alpha \beta \gamma} G_{\mu \nu \sigma \tau} A_{\alpha \beta \gamma} \\
& -\frac{1}{24} e^{-\phi} G_{\mu \nu \rho \tau} F_{\kappa \lambda}{ }^{a}{ }_{b} A_{\chi}{ }^{b}{ }_{a} \epsilon^{\mu \nu \rho \tau \kappa \lambda \chi}-V(\phi) .
\end{aligned}
$$

Compared to [24] we use the same conventions for the Riemann tensor but Hawking and Ellis conventions for the Ricci tensor and scalar. We have also rescaled $\phi \rightarrow \sqrt{5} \phi$ and the forms by $F \rightarrow \sqrt{2} e^{\phi} F, G \rightarrow \sqrt{2} e^{-2 \phi} G, A_{(1),(3)} \rightarrow \sqrt{2} A_{(1),(3)}$. The potential is given by

$$
V(\phi)=-60 m^{2}+10\left(m^{\prime}\right)^{2}
$$

where $m$ is a function of the single scalar field $\phi$,

$$
m=-\frac{2}{5} h e^{-4 \phi}-\frac{1}{10} g e^{\phi},
$$

with $g$ the gauge coupling (we have rescaled the coupling in [24] by $g \rightarrow g / \sqrt{2}$ ) and $h$ the (constant) topological mass. The supersymmetry variations of the fermions are given by

$$
\begin{aligned}
\delta \lambda^{a} & =\frac{\sqrt{5}}{2} \Gamma^{\mu} D_{\mu} \phi \epsilon^{a}+\frac{i}{2 \sqrt{5}} \Gamma^{\mu \nu} F_{\mu \nu}{ }_{b}{ }_{b} \epsilon^{b}+\frac{1}{24 \sqrt{5}} \Gamma^{\mu \nu \rho \tau} G_{\mu \nu \rho \tau} \epsilon^{a}-\sqrt{5} m^{\prime} \epsilon^{a}, \\
\delta \psi_{\mu}^{a} & =D_{\mu} \epsilon^{a}-\frac{i}{10}\left(\Gamma_{\mu}{ }^{\nu \rho}-8 \delta_{\mu}^{\nu} \Gamma^{\rho}\right) F_{\nu \rho}{ }_{b} \epsilon^{b}+\frac{1}{80}\left(\Gamma_{\mu}{ }^{\alpha \beta \gamma \delta}-\frac{8}{3} \delta_{\mu}^{\alpha} \Gamma^{\beta \gamma \delta}\right) G_{\alpha \beta \gamma \delta} \epsilon^{a} \\
& +m \Gamma_{\mu} \epsilon^{a}-i g A_{\mu b} \epsilon^{b},
\end{aligned}
$$

and the parameter $\epsilon^{a}$ is a symplectic-Majorana spinor, whose properties are summarized in appendix $\mathrm{A}$.

Let us introduce the following notation. Let $A_{p}, B_{q}$ be $p$ - and $q$-forms respectively. Then

$$
A\lrcorner B_{a_{1} \ldots a_{q-p}}=\frac{1}{p !} A^{b_{1} \ldots b_{p}} B_{b_{1} \ldots b_{p} a_{1} \ldots a_{q-p}} .
$$


The equations of motion and Bianchi identities are

$$
\begin{aligned}
d\left(e^{-2 \phi} G\right) & =0, \\
d\left(e^{\phi} F^{A}\right) & =g \epsilon^{A B C} e^{\phi} F^{B} \wedge A^{C}, \\
P & \left.\left.=5 \nabla^{2} \phi-4 G\right\lrcorner G+F^{A}\right\lrcorner F^{A}-V^{\prime}=0, \\
Q & =\star\left(e^{-2 \phi} d \star\left(e^{2 \phi} G\right)-\frac{1}{2} F^{A} \wedge F^{A}+8 h e^{-4 \phi} G\right)=0, \\
R^{A} & =\star\left(e^{\phi} d \star\left(e^{-\phi} F^{A}\right)-g \epsilon^{A B C} e^{\phi} \star F^{B} \wedge A^{C}-2 F^{A} \wedge G\right)=0, \\
E_{\mu \nu} & =R_{\mu \nu}-\frac{1}{3}\left(G_{\mu \alpha \beta \gamma} G_{\nu}{ }^{\alpha \beta \gamma}-\frac{1}{10} g_{\mu \nu} G_{\alpha \beta \gamma \delta} G^{\alpha \beta \gamma \delta}\right)-5 \partial_{\mu} \phi \partial_{\nu} \phi \\
& -\left(F_{\mu \alpha}^{A} F_{\nu}^{A \alpha}-\frac{1}{10} g_{\mu \nu} F_{\alpha \beta}^{A} F^{A \alpha \beta}\right)-\frac{2}{5} g_{\mu \nu} V=0 .
\end{aligned}
$$

When $h=0$, the theory lifts on an $S^{3}$ to the NS sector in $d=10$ [26]. When $h g>0$, it lifts on an $S^{4}$ to $d=11$ [27]. When $h \neq 0$, there is a subtlety in imposing the four-form field equation. The reason is that the 3 -form $A_{(3)}$, which is massive, would have twenty on-shell degrees of freedom if it satisfied an ordinary second order field equation. However the 3 -form in the $7 \mathrm{~d}$ supergravity multiplet should have only ten on-shell degrees of freedom. This is achieved by imposing the odd-dimensional selfduality equation [28]:

$$
e^{2 \phi} \star G-\frac{1}{2}\left(e^{\phi} F^{A} \wedge A^{A}-\frac{g}{6} \epsilon^{A B C} A^{A} \wedge A^{B} \wedge A^{C}\right)+8 h A_{(3)}=0 .
$$

Note that the exterior derivative of this equation is just $\star Q$. Imposing the Bianchi identity and $Q=0$ fixes $A_{(3)}$ up to an arbitrary closed three form. The closed three form is then determined by demanding that $A_{(3)}$ satisfies (2.13). In the examples given below, we will explicitly impose the Bianchi identity and $Q=0$, but leave the determination of the closed three form in $A_{(3)}$ implicit.

\section{Configurations admitting at least one null Killing spinor}

\subsection{The G-structure}

It is instructive to verify, using the standard formalism, that a single null spinor in seven dimensions defines an $\left(S U(2) \ltimes \mathbb{R}^{4}\right) \times \mathbb{R}$ structure. It was shown in 19 that we can define the following spinor bilinears

$$
\begin{aligned}
f^{(a b)} & =\bar{\epsilon}^{a} \epsilon^{b}, \\
\epsilon^{a b} V_{\mu} & =\bar{\epsilon}^{a} \Gamma_{\mu} \epsilon^{b}, \\
\epsilon^{a b} I_{\mu \nu} & =\bar{\epsilon}^{a} \Gamma_{\mu \nu} \epsilon^{b}, \\
\Omega_{\mu \nu \rho}^{(a b)} & =\bar{\epsilon}^{a} \Gamma_{\mu \nu \rho} \epsilon^{b} .
\end{aligned}
$$


From the reality properties of the gamma matrices and the symplectic Majorana condition, the vector $V_{\mu}$ and the two-form $I_{\mu \nu}$ are seen to be real, while instead the scalars and the 3-form can be rewritten as

$$
\begin{aligned}
f_{b}^{a} & =-i g^{A}\left(T^{A}\right)^{a}{ }_{b}, \\
\Omega^{a}{ }_{b} & =-i X^{A}\left(T^{A}\right)^{a}{ }_{b},
\end{aligned}
$$

with $g^{A}, X_{\mu \nu \rho}^{A}, A=1,2,3$, real. $\left(T^{A}\right)^{a}{ }_{b}=1 / 2\left(\sigma^{A}\right)^{a}{ }_{b}$ are generators of the $S U(2)$ Lie algebra, $\sigma^{A}$ being the Pauli matrices, and obey

$$
\left(T^{A}\right)^{a}{ }_{b}\left(T^{B}\right)^{b}{ }_{c}=\frac{1}{4} \delta^{A B} \delta_{c}^{a}+\frac{i}{2} \epsilon^{A B C}\left(T^{C}\right)^{a}{ }_{c} .
$$

One important consequence of the Fierz identity (A.11) is that $V_{\mu}$ is either timelike or null

$$
V^{2}=-\frac{1}{4} g^{A} g^{A}
$$

The case of a single timelike spinor has been analysed in a previous work [19]. Here we want to identify the G-structure associated a single null spinor, a spinor such that $g^{A}=0$. From the Fierz identities we may deduce

$$
\begin{aligned}
i_{V} I & =0, \\
I\lrcorner I & =0 \\
I_{\mu \tau} I^{\nu \tau} & =V_{\mu} V^{\nu} .
\end{aligned}
$$

These imply that

$$
I=V \wedge K, \quad K^{2}=1, \quad .
$$

Furthermore from the Fierz identities we may deduce the projection

$$
K_{\mu} \Gamma^{\mu} \epsilon^{a}=\epsilon^{a},
$$

together with

$$
i_{V} X^{A}=i_{K} X^{A}=0
$$

Let us introduce a null orthonormal basis where

$$
\begin{aligned}
V & =-e^{+}, \quad K=e^{5}, \\
d s^{2} & =-2 e^{+} e^{-}+\delta_{i j} e^{i} e^{j}+\left(e^{5}\right)^{2},
\end{aligned}
$$

$i, j=1, . ., 4$, and we choose an orientation

$$
\epsilon^{+-12345}=1
$$

Then from the seven dimensional duality relation for the gamma matrices, (3.13) becomes a chirality projection in the six Lorentzian dimensions orthogonal to $K$. It 
is well known [29] that a pair of symplectic Majorana-Weyl spinors (or a single Weyl spinor) defines an $S U(2) \ltimes \mathbb{R}^{4}$ structure in six Lorentzian dimensions, so our seven dimensional structure is simply $\left(S U(2) \ltimes \mathbb{R}^{4}\right) \times \mathbb{R}$. Now from the results of [5], we may immediately read off the form of the $X^{A}$ :

$$
\begin{aligned}
X^{A} & =2 e^{+} \wedge J^{A}, \\
J^{A i}{ }_{j}^{B j}{ }_{k} & =\epsilon^{A B C} J_{k}^{C i}-\delta^{A B} \delta_{k}^{i} .
\end{aligned}
$$

With our choice of conventions, the $J^{A}$ are selfdual on the base with orientation

$$
\epsilon^{i j k l}=\epsilon^{+-i j k l 5}
$$

The single spinor obeys the projection (3.13) and

$$
J_{i j}^{A} \Gamma^{i j} \epsilon^{a}=8 i T_{b}^{A a} \epsilon^{b} .
$$

We may completely fix the spinor by requiring that in addition to (3.13) it obeys the projections

$$
\begin{aligned}
& \Gamma^{12} \epsilon^{1}=i \epsilon^{2}, \\
& \Gamma^{13} \epsilon^{1}=-\epsilon^{2}, \\
& \Gamma^{14} \epsilon^{1}=i \epsilon^{1},
\end{aligned}
$$

so that the $J^{A}$ take the canonical form

$$
J^{1}=e^{12}+e^{34}, \quad J^{2}=-e^{13}+e^{24}, \quad J^{3}=e^{14}+e^{23} .
$$

For performing the calculations of the constraints it is very useful to have a set of projections satisfied by $\epsilon$ which are implied by the defining projections. We give such a set in appendix B.

\subsection{Constraints for supersymmetry}

Now we solve the constraints $\delta \lambda=\delta \psi_{\mu}=0$ for the single spinor $\epsilon$. In order to reduce as much as possible the computation required we will work with $\star G$, the dual of the four form $G$. Hopefully without risk of confusion, we also denote the dual by $G$. Where ambiguity can arise we will explicitly indicate the rank of the form. In terms of the dual $G$, the supersymmetry variations of the fermions are

$$
\begin{aligned}
\sqrt{5} \delta \lambda^{a} & =\frac{5}{2} \partial_{\mu} \phi \Gamma^{\mu} \epsilon^{a}+\frac{i}{2} F_{\mu \nu}^{A} \Gamma^{\mu \nu} T_{b}^{A a} \epsilon^{b}-\frac{1}{6} G_{\mu \nu \sigma} \Gamma^{\mu \nu \sigma} \epsilon^{a}-5 m^{\prime} \epsilon^{a} \\
\delta \psi_{\mu}^{a} & =\nabla_{\mu} \epsilon^{a}-\frac{i}{10}\left(\Gamma_{\mu}{ }^{\nu \sigma}-8 \delta_{\mu}^{\nu} \Gamma^{\sigma}\right) F_{\nu \sigma}^{A} T^{A a} \epsilon^{b}-\frac{3}{20} G_{\mu \nu \sigma} \Gamma^{\nu \sigma} \epsilon^{a}+\frac{1}{30} G_{\nu \sigma \tau} \Gamma_{\mu}{ }^{\nu \sigma \tau} \epsilon^{a} \\
& -i g A_{\mu}^{A} T^{A a} \epsilon^{b}+m \Gamma_{\mu} \epsilon^{a} .
\end{aligned}
$$




\subsubsection{Constraints from $\delta \lambda=0$}

Consider first the supersymmetry variation of $\delta \lambda$. We will in fact use two shortcuts from a previous work [19]. The first is that $i_{V} \partial \phi=0$. Also, on the assumption that $\epsilon$ is Killing, we have that $i_{V} F^{A}=0$. Thus

$$
\begin{aligned}
& \partial_{-} \phi=0, \\
& F_{-\mu}^{A}=0 .
\end{aligned}
$$

An implication of these is that the only place terms with a single $\Gamma^{-}$can arise in $\delta \lambda$ is in $G_{\mu \nu \sigma} \Gamma^{\mu \nu \sigma}$. Also, all terms with a single $\Gamma^{+}$drop out, since $\Gamma^{+} \epsilon=0$. Consider the $\Gamma^{-}$terms. These may easily be computed to be

$$
-\Gamma^{-}\left[G_{-i 5} \Gamma^{i}+i G_{-i j} J^{A i j} T^{A}\right] \epsilon .
$$

By linear independence, each of these must vanish separately. Hence

$$
\begin{aligned}
G_{-i 5} & =0, \\
G_{-i j}^{(+)} & =0 .
\end{aligned}
$$

Now all remaining terms in $\delta \lambda=0$ may be converted into terms containing no gamma matrices or a single gamma matrix on the base. The terms with a single gamma matrix are given by

$$
\left[\frac{5}{2} \partial_{i} \phi+G_{+-i}+\frac{1}{6} \epsilon_{i}{ }^{j k l} G_{j k l}+\frac{1}{2} F_{j 5}^{A} J^{A j}{ }_{i}\right] \Gamma^{i} \epsilon,
$$

and each component of the one form in square brackets must vanish. Finally, the terms involving no gamma matrices are of the form $\left(A+i \sum_{A} B^{A} T^{A}\right) \epsilon$, and by linear independence we must have $A=B^{A}=0$. This implies that

$$
\begin{aligned}
G_{+-5}+\frac{5}{2} \partial_{5} \phi-5\left(m+2 h e^{-4 \phi}\right) & =\frac{1}{4} F_{i j}^{A} J^{A i j}, \\
G_{5 i j}^{(+)} & =-\frac{1}{8} \epsilon^{A B C} F_{k l}^{A} J^{B k l} J_{i j}^{C} .
\end{aligned}
$$

This completes the analysis of $\delta \lambda^{(i)}=0$.

\subsection{2 $\delta \psi_{\mu}=0$}

Next we turn to the analysis of the Killing spinor equation. We will use another result from [19], namely that for null Killing spinors,

$$
\nabla V=i_{V} G-d \phi \wedge V-4 h e^{-4 \phi} I,
$$

so that $V$ is a Killing vector and

$$
\omega_{\mu \nu-}=G_{\mu \nu-}+\left(d \phi \wedge e^{+}\right)_{\mu \nu}+4 h e^{-4 \phi}\left(e^{+} \wedge e^{5}\right)_{\mu \nu} .
$$


Also we wish to comment on the choice of gauge. We always can, and will, choose the gauge $i_{V} A^{A}=0$. We also know that $i_{V} F^{A}=0$. Therefore if $v$ is the coordinate along the integral curves of $V$, we have that $A_{\mu}^{A}$ is independent of $v$, in the gauge $i_{V} A^{A}=0$. Therefore since we still have the freedom to perform $v$-independent gauge transformations, we may always choose a gauge such that

$$
A_{+}^{A}=A_{-}^{A}=0,
$$

and this is the gauge we work in henceforth. Now we know that each spacetime component of the Killing spinor equation can be reduced to the form (1.5) given in the introduction. All terms involving a single $\Gamma^{+}$drop out. Furthermore, we have explicitly checked that all the terms of the form

$$
\left(q_{\mu}+\Gamma^{-}\left(r_{\mu}+i r_{\mu}^{A} T^{A}+r_{\mu i} \Gamma^{i}\right)\right) \epsilon
$$

vanish identically as a consequence of the constraints we have already derived. Thus the Killing spinor equation reduces to

$$
\delta \psi_{\mu}=\left(i q_{\mu}^{A} T^{A}+q_{\mu i} \Gamma^{i}\right) \epsilon .
$$

Using the projections of appendix $\mathrm{B}$, it is easy to see that the spin connection components $\omega_{\mu i 5}$ contribute only to the $q_{\mu i} \Gamma^{i} \epsilon$ terms, the $\omega_{\mu i j}^{(-)}$components drop out, and the $\omega_{\mu i j}^{(+)}$components contribute only to the $i q_{\mu}^{A} T^{A} \epsilon$ terms. The surviving components of the spin connection may be fixed in terms of the fluxes by demanding that the linearly independent terms (3.38) vanish. Thus, from the + component of $\delta \psi_{\mu}=0$ we find

$$
\begin{aligned}
\omega_{+i j}^{(+)} & =G_{+i j}^{(+)}-\frac{1}{2} F_{+5}^{A} J_{i j}^{A}, \\
\omega_{+i 5} & =G_{+i 5}-F_{+j}^{A} J^{A j}{ }_{i} .
\end{aligned}
$$

From the - component we deduce

$$
\begin{aligned}
& \omega_{-i j}^{(+)}=0, \\
& \omega_{-i 5}=0 .
\end{aligned}
$$

The 5 component gives

$$
\begin{aligned}
& \omega_{5 i j}^{(+)}=G_{5 i j}^{(+)}+\frac{g}{2} A_{5}^{A} J_{i j}^{A}, \\
& \omega_{55 i}=4 \partial_{i} \phi+2 G_{+-i}+\frac{1}{3} \epsilon_{i j k l} G^{j k l} .
\end{aligned}
$$

Finally from the $i$ component we get

$$
\begin{aligned}
\omega_{i j 5} & =\left(G_{+-5}+\frac{3}{2} \partial_{5} \phi-5 m-6 h e^{-4 \phi}\right) g_{i j}+G_{5 i j}^{(-)}-G_{5 i j}^{(+)} \\
& -F_{i k}^{A(-)} J^{A k}{ }_{j} \\
\omega_{i j k} J^{A j k} & =2 g A_{i}^{A}-F_{i 5}^{A}+\epsilon^{A B C} F_{j 5}^{B} J^{C j}{ }_{i} \\
& -\left(3 \partial_{j} \phi+G_{+-j}\right) J^{A j}{ }_{i}
\end{aligned}
$$


This concludes the analysis of $\delta \psi_{\mu}=0$. The practical advantages of calculating the necessary and sufficient conditions for supersymmetry in this fashion are obvious. From the integrability conditions of Appendix D, we may deduce that it is sufficient to impose the Bianchi identity for the Yang-Mills fields and $G_{(4)}$, the field equation for $G_{(4)}$, the + component of the Yang-Mills field equation and the ++ component of the Einstein equation on this supersymmetric ansatz. All other field equations are satisfied identically.

\subsection{Introducing coordinates}

In this subsection we will introduce coordinates for our problem. Our null Killing spinor induces a natural coordinate system in which the associated symmetries of the metric are manifest. Closely following [10], we introduce coordinates $\left(v, u, z, x^{M}\right)$ such that the vectors dual to the basis one forms are

$$
\begin{aligned}
e^{+} & =-\frac{\partial}{\partial v}, \\
e^{-} & =-\frac{L \mathcal{F}}{2} \frac{\partial}{\partial v}+L \frac{\partial}{\partial u}, \\
e^{5} & =-\frac{B}{C} \frac{\partial}{\partial v}+\frac{1}{C} \frac{\partial}{\partial z}, \\
e^{i} & =e^{i \mu} \partial_{\mu} .
\end{aligned}
$$

Inverting we get

$$
\begin{aligned}
e^{+} & =-L^{-1}(d u+\lambda), \\
e^{-} & =d v+\frac{1}{2} \mathcal{F} d u+B d z+\nu, \\
e^{5} & =C(d z+\sigma), \\
e^{i} & =e_{M}^{i} d x^{M} .
\end{aligned}
$$

We have in fact fixed some of the gauge freedom in the metric. Now repeating an argument given in [10], we can deduce that $L, \mathcal{F}, B, C, \lambda, \nu, \sigma$ and $e_{M}^{i}$ may all be taken to be independent of $v$. The components of the spin connection for this metric were computed in [10]. We have performed the trivial modification to adjust to our slightly different conventions, and we give the result in appendix $\mathrm{C}$, where we use the following notation. Let $Q$ be a q-form on the base, satisfying $\mathcal{L}_{V} Q=0$ :

$$
Q=\frac{1}{q !} Q\left(u, z, x^{M}\right)_{M_{1} \ldots M_{q}} d x^{M_{1}} \wedge \ldots \wedge d x^{M_{q}} .
$$

Define the exterior derivative restricted to the base

$$
\tilde{d} Q \equiv \frac{1}{q !} \frac{\partial}{\partial x^{M_{1}}} Q_{M_{2} \ldots M_{q+1}} d x^{M_{1}} \wedge \ldots \wedge d x^{M_{q+1}},
$$


and denote the Lie derivatives with respect to $\frac{\partial}{\partial u}$ and $\frac{\partial}{\partial z}$ acting on such forms by $\partial_{u} Q, \partial_{z} Q$ respectively. Then, defining

$$
\mathcal{D} Q \equiv \tilde{d} Q-\sigma \wedge \partial_{z} Q+\lambda \wedge \partial_{u} Q
$$

we have

$$
d Q=\mathcal{D} Q-L e^{+} \wedge \partial_{u} Q+C^{-1} e^{5} \wedge \partial_{z} Q
$$

Finally we define the following quatities:

$$
\begin{aligned}
M_{i j} & =\delta_{i k}\left(\partial_{u} e^{k}\right)_{j}, \\
\Lambda_{i j} & =\delta_{i k}\left(\partial_{z} e^{k}\right)_{j} .
\end{aligned}
$$

From the expression for the spin connection components in appendix $\mathrm{C}$, we may rewrite the constraints $\omega_{-i 5}=\omega_{-i j}^{(+)}=0$ as

$$
\begin{aligned}
\lambda & =\lambda(u, x), \\
\mathcal{D} \lambda^{(+)} & =0 .
\end{aligned}
$$

\subsection{Refining the classification}

In this section we have given the full set of neccessary and sufficient conditions for the existence of a single arbitrary null spinor. In the next four sections we will illustrate the computation of the constraints associated with the existence of additional spinors, for each of the four possibilities for the structure groups. As discussed in the introduction, this involves computing the expressions

$$
\begin{aligned}
& {\left[\Delta_{\lambda}, Q\right] \epsilon=0,} \\
& {\left[\mathcal{D}_{\mu}, Q\right] \epsilon=0,}
\end{aligned}
$$

for a choice of $Q$ compatible with the desired G-structure, and reducing them to canonical form, as a manifest sum of basis spinors. As has been stated, to do this for the most general choices of $Q$ requires a lot of computation, and we will not perform the refined classification in full generality. For the structure groups $S U(2)$, $\mathbb{R}^{5}$ and the identity, we will restrict attention to illustrative examples, for particular choices of $Q$. This means that we will make specific ansätze for the additional Killing spinors. Also for computational convenience, unless otherwise stated we will restrict attention to bosonic configurations for which the Yang-Mills fields are of the form

$$
\begin{aligned}
A^{A} & =A_{i}^{A}(x) e^{i}, \\
F^{A} & =\frac{1}{2} F_{i j}^{A}(x) e^{i} \wedge e^{j},
\end{aligned}
$$

but which are otherwise general. Note that this is the only assumption we make about the form of the bosonic fields; all the additional constraints we derive will follow as a consequence of the choice of $Q$. 


\section{4. $\left(S U(2) \ltimes \mathbb{R}^{4}\right) \times \mathbb{R}$ structure}

As we saw in the introduction, the simplest additional Killing spinors to incorporate are those which share the same isotropy group as the fiducial Killing spinor, and so imply no further reduction of the structure group. These spinors are parameterised by four real functions; given our assumption of the form of the Yang-Mills fields, we will completely analyse all $\left(S U(2) \ltimes \mathbb{R}^{4}\right) \times \mathbb{R}$ structures with more than one Killing spinor. In the first subsection we will derive the constraints, and in the second subsection we will construct a class of solutions for which the structure group is strictly $\left(S U(2) \ltimes \mathbb{R}^{4}\right) \times \mathbb{R}$ and not some subgroup.

\subsection{Constraints}

Consider an arbitrary $(+,+)$ spinor, which we denote by

$$
\left(\delta+i \delta^{A} T^{A}\right) \epsilon .
$$

As discussed in the introduction, assuming that this spinor is Killing does not imply any reduction of the structure group, but it does yield additional constraints on the intrinsic torsion. The commutators (3.63) for such spinors are easy to compute, since they just pick out the Yang-Mills terms in the supersymmetry variations. First consider

$$
\left[\Delta_{\lambda}, \delta+i \delta^{A} T^{A}\right] \epsilon=-\frac{1}{2} F_{i j}^{A} \Gamma^{i j} \delta^{B}\left[T^{A}, T^{B}\right] \epsilon .
$$

Reducing to canonical form, we get

$$
\left(\frac{1}{4} \epsilon^{A B C} F_{i j}^{A} \delta^{B} J^{C i j}+\frac{i}{2}\left(F_{i j}^{B} J^{B i j} \delta^{A}-F_{i j}^{A} J^{B i j} \delta^{B}\right) T^{A}\right) \epsilon .
$$

Next consider the Killing spinor equation. The commutator is

$$
\left[\nabla_{\mu}-i\left(g A_{\mu}^{A}+\frac{1}{10}\left(\Gamma_{\mu \nu \sigma}-8 g_{\mu \nu} \Gamma_{\sigma}\right) F^{A \nu \sigma}\right) T^{A}, \delta+i \delta^{B} T^{B}\right] \epsilon .
$$

The - component is particularly easy to evaluate. It reduces to

$$
\left(\partial_{-} \delta+i \partial_{-} \delta^{A} T^{A}\right) \epsilon
$$

The + component is

$$
\left(\partial_{+} \delta+i \partial_{+} \delta^{A} T^{A}+\frac{1}{5} \Gamma^{-}\left[\Delta_{\lambda}, i \delta^{A} T^{A}\right]\right) \epsilon .
$$

The 5 component is

$$
\left(\partial_{5} \delta-\frac{1}{5}\left[\Delta_{\lambda}, i \delta^{A} T^{A}\right]+i \partial_{5} \delta^{A} T^{A}\right) \epsilon,
$$

and for the $i$ component we have

$$
\left.\left[\partial_{i} \delta+i\left(\partial_{i} \delta^{A}+g \epsilon^{A B C} A_{i}^{B} \delta^{C}\right) T^{A}\right)-\frac{1}{2} \epsilon^{A B C}\left(\frac{3}{5} F_{i j}^{A(+)}+F_{i j}^{A(-)}\right) \delta^{B} J_{k}^{C j} \Gamma^{k}\right] \epsilon .
$$


Now, requiring the vanishing of the commutators acting on $\epsilon$ implies the following algebraic restrictions on the Yang-Mills fields in terms of the parameters $\delta^{A}$ :

$$
\begin{aligned}
\epsilon^{A B C} F_{i j}^{A} \delta^{B} J^{C i j} & =0, \\
\delta^{A} F_{i j}^{B} J^{B i j}-\delta^{B} F_{i j}^{A} J^{B i j} & =0, \\
\epsilon^{A B C} F_{j k}^{A(-)} \delta^{B} J^{C j}{ }_{i} & =0 .
\end{aligned}
$$

We also get a set of differential constraints on the $\delta, \delta^{A}$ which are

$$
\begin{aligned}
\partial_{\mu} \delta & =0, \\
\partial_{-} \delta^{A}=\partial_{+} \delta^{A} & =\partial_{5} \delta^{A}=0, \\
\partial_{i} \delta^{A} & =-g \epsilon^{A B C} A_{i}^{B} \delta^{C} .
\end{aligned}
$$

Since we have already assumed that $\epsilon$ is Killing, and we have derived that $\delta=$ const, we take $\delta=0$. We also have

$$
\delta^{A}=\delta^{A}(x)
$$

Given the Yang-Mills Bianchi identity, the integrability condition for (4.11) implies that $\epsilon^{A B C} F_{i j}^{B} \delta^{C}=0$, and so implies the algebraic constraints. Therefore a bosonic configuration admitting the Killing spinor $\epsilon$ also admits the Killing spinor $i \delta^{A} T^{A} \epsilon$ if and only if equation (4.11) is satisfied. By performing an $x$ dependent $S U(2)$ gauge transformation (thus preserving $A_{+}^{A}=A_{-}^{A}=A_{5}^{A}=0$ ) we may set

$$
\delta^{A}=k \delta^{1 A}
$$

for some constant $k$ which can be eliminated by a constant rescaling, and so the existence of this Killing spinor implies and is implied by the condition that the Yang-Mills field is truncated to a $U(1)$ subgroup,

$$
A^{2}=A^{3}=0
$$

Clearly, requiring the existence of a third linearly independent $(+,+)$ Killing spinor would imply that

$$
A^{1}=0
$$

and then the configuration automatically admits a fourth linearly independent spinor. Thus, the $\left(S U(2) \ltimes \mathbb{R}^{4}\right) \times \mathbb{R}$ structure can admit one, two, or four linearly independent Killing spinors, depending on whether the Yang-Mills fields are $S U(2), U(1)$ or zero. Incorporating additional $(+,+)$ spinors implies no further constraints on $G, \phi$ or $\omega$, beyond those derived in section 2. From these results, we also see that when the Yang-Mills fields are truncated to a $U(1)$ subgroup, Killing spinors always come in pairs; if $\epsilon_{K}$ is Killing, then so is the linearly independent spinor $i T^{1} \epsilon_{K}$, since $T^{1}$ commutes with the operators in the fermion supersymmetry variations. Similarly, when the Yang-Mills fields vanish, Killing spinors always come in groups of four. 


\subsection{Examples}

Let us now present a family of solutions for which the structure group is strictly $\left(S U(2) \ltimes \mathbb{R}^{4}\right) \times \mathbb{R}$ and not some subgroup. It is easy to verify that the vacuum solution

$$
d s^{2}=(4 h z)^{-2}\left(-d t^{2}+d r^{2}+d z^{2}+d s^{2}\left(\mathcal{M}_{4}\right)\right),
$$

where $\mathcal{M}_{4}$ is hyperkähler and $16 h=g$, satisfies all the constraints of section 2, and the ++ component of the Einstein equations is satisfied. Since the YangMills fields vanish, these solutions admit Killing spinors in multiples of four. When $\mathcal{M}_{4}=\mathbb{R}^{4}$, this is nothing but the maximally supersymmetric $A d S_{7}$ solution of the theory. Choosing any other hyperkähler base reduces the number of independent Killing spinors to four. One may show that demanding the existence of an arbitrary additional Killing spinor of the form (1.13) implies the constraints

$$
\begin{aligned}
\tilde{\nabla}_{i} \delta_{j} & =0, \\
\partial_{\mu} \theta & =\partial_{\mu} \theta^{A}=0, \\
\tilde{\nabla}_{i} \theta_{j} & =2 m \theta \delta_{i j}+m \theta^{A} J_{i j}^{A},
\end{aligned}
$$

where $\tilde{\nabla}$ denotes the Levi-Civita connection on the base. Given that for vanishing Yang-Mills fields Killing spinors come in linearly independent groups of four, the existence of a single one form $\delta_{i}$ satisfying (4.17) implies the existence of three more linearly independent solutions, and thus (4.17) implies that either $\delta_{i}=0$ or the base is flat. Similarly, since $\theta, \theta^{A}$ are constant and $\tilde{\nabla} J^{A}=0$, (4.19) implies that $\left[\tilde{\nabla}_{i}, \tilde{\nabla}_{j}\right] \theta_{k}=0$, and the existence of one one form on the base satisfying this equation implies the existence of three more linearly independent solutions. Hence either $\theta_{j}=0$ or the base is flat. If the base is not flat, then we must have $\delta_{i}=\theta_{i}=0$

and, given $m \neq 0$, that $\theta=\theta^{A}=0$. Thus the solutions (4.16) preserve precisely four supersymmetries, with a strictly $\left(S U(2) \ltimes \mathbb{R}^{4}\right) \times \mathbb{R}$ structure, when the base is a non-flat hyperkähler manifold.

\section{5. $S U(2)$ structure}

In this section we will study configurations in the theory admitting an $S U(2)$ structure. We will first derive the constraints for the existence of a particular choice of additional Killing spinor. We will then derive neccessary and sufficient conditions for the existence of eight Killing spinors fixed by the same $S U(2)$. Finally, we will discuss some illustrative explicit solutions.

\subsection{One additional Killing spinor}

Consider an arbitrary linear combination of $(+,+)$ and $(-,+)$ spinors, which we denote by

$$
\left(\delta+i \delta^{A} T^{A}+\Gamma^{-}\left(\theta+i \theta^{A} T^{A}\right)\right) \epsilon
$$


and where at least one of the $\theta, \theta^{A} \neq 0$. To derive the constraints following from $\delta \lambda=0$ for this spinor, we must impose

$$
\left[\Delta_{\lambda}, \delta+i \delta^{A} T^{A}+\Gamma^{-}\left(\theta+i \theta^{A} T^{A}\right)\right] \epsilon=0 .
$$

We have already calculated the $\left[\Delta_{\lambda}, \delta+i \delta^{A} T^{A}\right] \epsilon$ terms, in the previous section. Calculating the commutator

$$
\left[\Delta_{\lambda}, i \theta^{A} \Gamma^{-} T^{A}\right] \epsilon
$$

while entirely straightforward, is a tedious exercise. We will therefore restrict attention to spinors with $\theta^{A}=0$, but $\theta \neq 0$. The $\theta$ dependent terms in the commutator, reduced to canonical form, then become

$$
\begin{aligned}
{\left[\Delta_{\lambda}, \theta \Gamma^{-}\right] \epsilon } & =\theta\left[-5 \partial_{+} \phi+2 i J^{A i j} G_{+i j} T^{A}+2 G_{+i 5} \Gamma^{i}+2 G_{+-i} \Gamma^{-} \Gamma^{i}-5 \partial_{5} \phi \Gamma^{-}\right. \\
& \left.+2 i G_{5 i j} J^{A i j} \Gamma^{-} T^{A}\right] \epsilon
\end{aligned}
$$

We may immediately deduce

$$
G_{+i 5}=G_{+-i}=\partial_{5} \phi=G_{5 i j}^{(+)}=0 .
$$

Then from the terms in $\left[\Delta_{\lambda}, \delta+i \delta^{A} T^{A}\right] \epsilon$, and using $G_{5 i j}^{(+)}=0$, we obtain

$$
\begin{aligned}
\partial_{+} \phi & =0 \\
\theta G_{+i j} J^{A i j} & =-\frac{1}{4}\left(F_{i j}^{B} J^{B i j} \delta^{A}-F_{i j}^{A} J^{B i j} \delta^{B}\right) .
\end{aligned}
$$

Next, commuting the - component of the Killing spinor equation, we deduce

$$
\begin{aligned}
\partial_{-} \theta & =0, \\
\partial_{-} \delta & =-\theta\left(2\left(5 m+6 h e^{-4 \phi}\right)+\frac{1}{2} F_{i j}^{A} J^{A i j}\right), \\
\partial_{-} \delta^{A} & =0 .
\end{aligned}
$$

From the + component, we get

$$
\begin{aligned}
\partial_{+} \theta & =0, \\
\partial_{+} \delta & =\theta \omega_{+5+}, \\
\partial_{+} \delta^{A} & =0 \\
\omega_{++i} & =0 .
\end{aligned}
$$

The 5 component gives

$$
\begin{aligned}
\partial_{5} \theta & =0, \\
\partial_{5} \delta & =\theta \omega_{55+}, \\
\partial_{5} \delta^{A} & =0, \\
\omega_{5+i} & =0,
\end{aligned}
$$


and from the $i$ component, we get

$$
\begin{aligned}
\partial_{i} \theta & =-\theta \partial_{i} \phi, \\
\partial_{i} \delta & =0, \\
\partial_{i} \delta^{A} & =-g \epsilon^{A B C} A_{i}^{B} \delta^{C}, \\
\frac{1}{2} \epsilon^{A B C}\left(\frac{3}{5} F_{i k}^{A(+)}+F_{i k}^{A(-)}\right) \delta^{B} J_{j}^{C k} & =\theta\left(\frac{1}{5} G_{+i j}^{(+)}+G_{+i j}^{(-)}-\omega_{i j+}\right), \\
\left(\frac{1}{4} F_{k l}^{A} J^{A k l}+4 h e^{-4 \phi}\right) g_{i j} & =F_{i k}^{A(-)} J_{j}^{A k} .
\end{aligned}
$$

As before, (5.21) implies that $\epsilon^{A B C} F_{i j}^{A} \delta^{C}=0$. We may easily solve the differential equations for $\theta$ to find

$$
\theta=k e^{-\phi}
$$

for some nonzero constant $k$; by a constant rescaling of the spinor, we may take $k=1$. Furthermore, since $A_{i k}^{(+)} B_{j}^{(-) k}$ is in general symmetric and traceless on $i, j$, (5.23) implies that

$$
\begin{aligned}
F_{i j}^{A} J^{A i j} & =-16 h e^{-4 \phi}, \\
F_{i j}^{A(-)} & =0 .
\end{aligned}
$$

Summary Given the form (3.65) of the Yang-Mills fields, the following conditions, in addition to those of section 2, are necessary and sufficient for a bosonic configuration to admit the Killing spinors $\epsilon,\left(\delta+i \delta^{A} T^{A}+\theta \Gamma^{-}\right) \epsilon, \theta \neq 0$. The functions $\theta, \delta$ and $\delta^{A}$ are required to satisfy

$$
\begin{aligned}
\theta & =e^{-\phi}, \\
\partial_{-} \delta & =g, \\
\partial_{i} \delta & =0, \\
\delta^{A} & =\delta^{A}(x), \\
\partial_{i} \delta^{A} & =-g \epsilon^{A B C} A_{i}^{B} \delta^{C},
\end{aligned}
$$

The matter fields are constrained according to

$$
\begin{aligned}
\phi & =\phi(x), \\
G_{(3)} & =\left(4 h e^{-4 \phi}-\frac{g}{2} e^{\phi}\right) e^{+} \wedge e^{-} \wedge e^{5}+e^{+} \wedge P_{1}^{(-)} \\
& +e^{-} \wedge P_{2}^{(-)}+e^{5} \wedge P_{3}^{(-)}+\frac{5}{2} \star_{4} d \phi, \\
F^{A} & =\frac{1}{2} F_{i j}^{A(+)} e^{i} \wedge e^{j}, \\
F_{i j}^{A} J^{A i j} & =-16 h e^{-4 \phi}, \\
\epsilon^{A B C} F_{i j}^{B} J^{C i j} & =0 \\
\epsilon^{A B C} F_{i j}^{B} \delta^{C} & =0
\end{aligned}
$$


where $P_{1,2,3}^{(-)}$are arbitrary anti selfdual two forms on the base, and $\star_{4}$ denotes the Hodge dual on the base. Finally, there are the following constraints on the independent components of the spin connection:

$$
\begin{aligned}
\omega_{(\mu \nu)-}=\omega_{-5 i} & =\omega_{+5 i}=\omega_{++i}=0, \\
\omega_{-+5} & =-\frac{g}{2} e^{\phi}, \\
\omega_{-+i} & =\omega_{5 i 5}=\partial_{i} \phi \\
\omega_{+5+} & =\theta^{-1} \partial_{+} \delta \\
\omega_{55+} & =\theta^{-1} \partial_{5} \delta \\
\omega_{+i j} & =-\omega_{i j+}=-P_{1}^{(-)}, \\
\omega_{-i j} & =-P_{2}^{(-)}, \\
\omega_{5 i j} & =-\omega_{i j 5}=-P_{3}^{(-)}, \\
\omega_{i j k} J^{A j k} & =2 g A_{i}^{A}-3 \partial_{j} \phi J^{A j}{ }_{i},
\end{aligned}
$$

and the remaining nonzero components may be read off from the equalities in appendix C. It may be verified that it is sufficient to impose the Bianchi identities for the forms and the field equation for $G$, and all other equations of motion are implied by the existence of the pair of Killing spinors.

Clearly, demanding the existence of the second Killing spinor significantly reduces the complexity of the ansatz, and much more of the field content is fixed in terms of the structure. Before considering some explicit examples, let us now turn to the classification of all solutions admitting a strictly $S U(2)$ structure with eight supersymmetries.

\section{$5.2 S U(2)$ structures with eight supersymmetries}

In this subsection, we will classify all configurations admitting eight linearly independent Killing spinors sharing the common projection $\Gamma^{1234} \epsilon=\epsilon$ (note that this implies the common projection $\Gamma^{+-5} \epsilon=-\epsilon$ ). This is the maximal supersymmetry compatible with a strictly $S U(2)$ structure. We will perform the classification in full generality, and we thus relax the assumptions made about the form of the Yang-Mills fields for this subsection. First consider the vanishing of $\delta \lambda$. Since this is linear in $\epsilon$ and contains no derivatives, it must vanish when acting on each of the eight basis spinors individually. It is a simple matter to verify that this implies that the matter fields are restricted to be of the following form:

$$
\begin{aligned}
\phi & =\phi(x), \\
F^{A} & =\frac{1}{2} F_{i j}^{A(-)} e^{i} \wedge e^{j}, \\
G & =5\left(m+2 h e^{-4 \phi}\right) e^{+} \wedge e^{-} \wedge e^{5}+\frac{1}{2} G_{-i j}^{(-)} e^{-} \wedge e^{i} \wedge e^{j}+\frac{1}{2} G_{+i j}^{(-)} e^{+} \wedge e^{i} \wedge e^{j} \\
& +\frac{1}{2} G_{5 i j}^{(-)} e^{5} \wedge e^{i} \wedge e^{j}+\frac{5}{2} \star_{4} d \phi .
\end{aligned}
$$


The vanishing of $F_{+\mu}^{A}, F_{5 \mu}^{A}$ implies that locally we may choose a gauge such that $A^{A}=A_{i}^{A} e^{i}$. Thus, acting on any of the eight Killing spinors, the supercovariant derivative reduces to the following form:

$$
\begin{aligned}
\mathcal{D}_{-} & =\nabla_{-}+\frac{3}{2}\left(m+2 h e^{-4 \phi}\right) \Gamma^{+5}-\frac{1}{2} \partial_{i} \phi \Gamma^{+i}-m \Gamma^{+}, \\
\mathcal{D}_{+} & =\nabla_{+}-\frac{3}{2}\left(m+2 h e^{-4 \phi}\right) \Gamma^{-5}-\frac{1}{2} \partial_{i} \phi \Gamma^{-i}-m \Gamma^{-}, \\
\mathcal{D}_{5} & =\nabla_{5}+\frac{3}{2}\left(m+2 h e^{-4 \phi}\right) \Gamma^{+-}+\frac{1}{2} \partial_{i} \phi \Gamma^{5 i}+m \Gamma^{5}, \\
\mathcal{D}_{i} & =\nabla_{i}+i F_{i j}^{A} \Gamma^{j} T^{A}+\frac{1}{2}\left(G_{+i j} \Gamma^{+j}+G_{-i j} \Gamma^{-j}+G_{5 i j} \Gamma^{5 j}\right) \\
& +\left(m+2 h e^{-4 \phi}\right) \Gamma_{i}^{+-5}+\frac{1}{2} \partial_{i} \phi-i g A_{i}^{A} T^{A}+m \Gamma^{i} .
\end{aligned}
$$

By assumption, there exist four Killing spinors of the form $\left(\delta+i \delta^{A} T^{A}+\Gamma^{-}(\theta+\right.$ $\left.\left.i \theta^{A} T^{A}\right)\right) \epsilon$, with four distinct choices of the functions $\theta, \theta^{A}$, at least one of which is nonzero in each case, and none of which is zero for all four. Let us evaluate $\left[\mathcal{D}_{\mu}, Q\right] \epsilon$ for one of these Killing spinors. From the - component, we find the constraints

$$
\begin{aligned}
\partial_{-} \theta & =\partial_{-} \theta^{A}=0, \\
\partial_{-} \delta & =-2\left(5 m+6 h e^{-4 \phi}\right) \theta, \\
\partial_{-} \delta^{A} & =-2\left(5 m+6 h e^{-4 \phi}\right) \theta^{A} .
\end{aligned}
$$

Next, from the + component, we find

$$
\begin{aligned}
\partial_{+} \theta & =\partial_{+} \theta^{A}=\omega_{++i}=0, \\
\partial_{+} \delta & =-\theta \omega_{++5}, \\
\partial_{+} \delta^{A} & =-\theta^{A} \omega_{++5} .
\end{aligned}
$$

The 5 component gives

$$
\begin{aligned}
\partial_{5} \theta & =\partial_{5} \theta^{A}=\omega_{5+i}=0 \\
\partial_{5} \delta & =-\theta \omega_{5+5} \\
\partial_{5} \delta^{A} & =-\theta^{A} \omega_{5+5} .
\end{aligned}
$$

Finally, consider the $i$ component, and in particular, the constraints derived from the vanishing of the $\Gamma^{-i} \epsilon$ term. These read

$$
-\theta F_{i k}^{A} J_{j}^{A k}+\frac{1}{2} F_{i j}^{A} \theta^{A}+4 h \theta e^{-4 \phi} g_{i j}+2 h e^{-4 \phi} \theta^{A} J_{i j}^{A}=0 .
$$

Recall that each $F_{i j}^{A}$ is anti selfdual. Then extracting the antisymmetric part of (5.35), and further decomposing in selfdual and anti selfdual parts, we find

$$
\begin{aligned}
F_{i j}^{A} \theta^{A} & =0, \\
h J_{i j}^{A} \theta^{A} & =0 .
\end{aligned}
$$


Since by assumption there exist three linearly independent Killing spinors with different nonzero $\theta^{A}$, these equations imply that $h=F^{A}=0$. Since the Yang-Mills field strengths vanish, we may locally choose the gauge so that also $A^{A}=0$. Then since each $T^{A}$ commutes with the operators in the supersymmetry variations, we see that we may take four of the Killing spinors to be $\epsilon, i T^{A} \epsilon$. It remains to determine the other four Killing spinors with nonzero $\theta, \theta^{A}$. Since $i T^{A}$ commute with the operators in the supersymmetry variations, once one of the Killing spinors, $\epsilon_{K}$, with nonzero $\theta, \theta^{A}$ is determined, the other three may be taken to be $i T^{A} \epsilon_{K}$. We thus only need to determine the single Killing spinor $\epsilon_{K}$. The remaining constraints from the $i$ component of the Killing spinor equation are

$$
\begin{aligned}
\partial_{i} \delta & =\partial_{i} \delta^{A}=0, \\
\partial_{i} \theta & =-\partial_{i} \phi \theta, \\
\partial_{i} \theta^{A} & =-\partial_{i} \phi \theta^{A}, \\
G_{i j+} & =\omega_{i j+} .
\end{aligned}
$$

We may easily solve for the differential equations for $\theta, \theta^{A}$ to find

$$
\begin{aligned}
\theta & =k e^{-\phi}, \\
\theta^{A} & =k^{A} e^{-\phi},
\end{aligned}
$$

for some constants $k, k^{A}$, at least one of which is nonzero. Now, note from the differential equations for $\delta, \delta^{A}$ that if $k=0$, then $\delta$ is constant, and if $k^{A}=0$, then $\delta^{A}$ is constant. These constants may be taken to be zero, since we are free to add to $\epsilon_{K}$ a constant multiple of the four Killing spinors $\epsilon, i T^{A} \epsilon$. Let us thus write

$$
\begin{aligned}
\delta & =k \delta^{\prime}, \\
\delta^{A} & =k^{A} \delta^{A \prime},
\end{aligned}
$$

with no sum on $A$ in the second of these equations. Now for each nonzero $k, k^{A}$, the associated $\delta^{\prime}, \delta^{A \prime}$ obey the same set of differential equations, which schematically are

$$
\partial_{\mu} f=B_{\mu} .
$$

Locally the solution exists and is unique, up to a constant which as before may be taken to be zero. Hence

$$
\begin{aligned}
\delta & =k f(u, v, z, x), \\
\delta^{A} & =k^{A} f(u, v, z, x) .
\end{aligned}
$$

We may easily fix the $v$ dependence of $f$ from equation (5.32), finding

$$
f(u, v, z, x)=g v+\hat{f}(u, z, x) .
$$


We must impose $\partial_{i} f=0$, but the function $\hat{f}$ is otherwise arbitrary. Making a choice for it determines $\omega_{++5}$ and $\omega_{5+5}$ through equations (5.33) and (5.34). Thus

$$
\epsilon_{K}=\left(k g v+k \hat{f}+i T^{A} k^{A} \hat{f}+e^{-\phi} \Gamma^{-}\left(k+i k^{A} T^{A}\right)\right) \epsilon,
$$

and the remaining three Killing spinors are $i T^{A} \epsilon_{K}$. By taking linear combinations with constant coefficients of these four spinors, we may in fact choose the set of four Killing spinors to be

$$
\epsilon_{K}^{\prime}=\left(g v+\hat{f}(u, z, x)+e^{-\phi} \Gamma^{-}\right) \epsilon,
$$

and $i T^{A} \epsilon_{K}^{\prime}$. We have thus determined all eight Killing spinors, and the necessary and sufficient conditions for their existence. It is intriguing to note that all eight may be generated by repeated application of the three matrices $i T^{1}, i T^{2}$ and $(g v+$ $\left.f^{\prime}(u, z, x)+e^{-\phi} \Gamma^{-}\right)$to $\epsilon$, and also that the third one of these matrices is precisely of the form we derived in the previous subsection, for a single additional Killing spinor. Perhaps for all configurations with an $S U(2)$ structure, the additional Killing spinors are of this form; that is, perhaps these are the only three matrices defining an $S U(2)$ structure which, together with their products, can commute with $\Delta_{\lambda}$ and $\mathcal{D}_{\mu}$, when acting on $\epsilon$. We have not verified this conjecture. However, if it is true that there are only a few matrices $Q$ that can generate additional Killing spinors $Q \epsilon$, then the problem of performing a fully refined classification will not be as formidable as it might appear. It will be interesting to explore this point in the future, and in other contexts. We will now turn to some explicit examples.

\subsection{Examples}

To illustrate the case of an $S U(2)$ structure, let us first see how known $A d S_{3} \times \mathcal{M}_{4}$ solutions of the theory arise in our formalism. This will also illustrate the effect of additional Killing spinors on the intrinsic torsion of the structure. We will then generalise the construction, to obtain new $A d S_{3}$ solutions.

\subsection{1 $A d S_{3} \times \mathcal{M}_{4}$ solutions}

Let us make the following ansatz for a second independent Killing spinor:

$$
\epsilon_{K}=\left(g v+\Gamma^{-}\right) \epsilon
$$

This choice solves all the differential constraints on $\theta, \delta$; also, it implies that $\omega_{+5+}=$ $\omega_{55+}=0$, and $\phi=$ constant; without loss of generality, when $\phi=$ constant, by constant rescalings of the forms and the couplings we may take $\phi=0$. We will take the metric to be of the direct product form

$$
d s^{2}=L^{-1}(z) d u d v+C^{2}(z) d z^{2}+h_{M N}(x) d x^{M} d x^{N} .
$$

For this choice of metric, the constraint $\omega_{-+5}=-g / 2$ is equivalent to

$$
\partial_{z} L=-g L C
$$


from Appendix C, which is solved by

$$
L=(g z)^{-2}, \quad C=2 L^{1 / 2},
$$

and so by rescaling $z$ the metric in the +-5 directions may be written as

$$
\frac{4}{g^{2}}\left(z^{2}\left(-d t^{2}+d y^{2}\right)+\frac{d z^{2}}{z^{2}}\right),
$$

which is the metric on $A d S_{3}$ with $A d S$ length $2 g^{-1}$. Our choice of metric implies that $P_{1,2,3}^{(-)}=0$, so the fluxes are

$$
\begin{aligned}
G_{(3)} & =\left(4 h-\frac{g}{2}\right) e^{+} \wedge e^{-} \wedge e^{5}, \\
F^{A} & =f^{A B} J^{B},
\end{aligned}
$$

where

$$
\begin{aligned}
f^{[A B]} & =0, \\
f^{A A} & =-4 h .
\end{aligned}
$$

Since $G$ is closed and coclosed, the Bianchi identity for $G_{(4)}$ is automatically satisfied, while the field equation for $G_{(4)}$ is

$$
-8 h\left(4 h-\frac{g}{2}\right)-f^{A B} f^{A B}=0 .
$$

We may rewrite the final constraint of (5.29) in covariant form as

$$
\nabla_{i} J_{j k}^{A}+g \epsilon^{A B C} A_{i}^{B} J_{j k}^{C}=0,
$$

or equivalently,

$$
A_{i}^{A}=-\frac{1}{8 g} \epsilon^{A B C} J^{B j k} \nabla_{i} J_{j k}^{C} .
$$

Imposing the Yang-Mills Bianchi identity then implies that

$$
f^{A B} J_{i j}^{B}=\frac{1}{2 g} J^{A k l} R_{k l i j} \equiv \frac{1}{g} \mathfrak{R}_{i j}^{A} .
$$

Taking the of commutator of two $S U(2)$ covariant derivatives acting on $J^{A}$ we may obtain

$$
J_{i}^{A k} R_{k j}=\mathfrak{R}_{i j}^{A}+\epsilon^{A B C} J_{i}^{B k} \mathfrak{R}_{k j}^{C},
$$

where $R_{i j}$ is the Ricci tensor, and hence that

$$
\begin{aligned}
R & =4 g f^{A A}=-16 h g, \\
R_{i j} & =\frac{R}{4} g_{i j},
\end{aligned}
$$

where $R$ is the scalar curvature of the base. Hence the Yang-Mills Bianchi identity implies that we must choose the base to be Einstein (with negative scalar curvature when $h g>0$ ). The Yang-Mills fields are then determined by (5.55), and once $f^{A B}$ is determined, (5.52) fixes $h$ in terms of $g$. There are no further constraints, and all remaining field equations are identically satisfied. 
Two Killing spinors Let us take the base to be $H^{4}$, equipped with the metric

$$
d s_{4}^{2}=\frac{4 \mu^{2}}{\left(1-r^{2}\right)^{2}}\left(d r^{2}+\frac{r^{2}}{4} \delta_{A B} \sigma^{A} \sigma^{B}\right),
$$

where the $\sigma^{A}$ are the right invariant one forms on an $S^{3}, d \sigma^{A}=-\frac{1}{2} \epsilon^{A B C} \sigma^{B} \sigma^{C}$. Let us choose the vierbeins to be

$$
e^{1}=\frac{\mu r}{1-r^{2}} \sigma^{1}, \quad e^{2}=\frac{\mu r}{1-r^{2}} \sigma^{3}, \quad e^{3}=\frac{\mu r}{1-r^{2}} \sigma^{2}, \quad e^{4}=-\frac{2 \mu}{1-r^{2}} d r .
$$

Then we may simply read off the full solution; we find that $g=\frac{28}{3} h, \mu^{2}=\frac{7}{g^{2}}$, and the bosonic fields are given by

$$
\begin{aligned}
d s^{2} & =\frac{1}{g^{2}} d s^{2}\left(A d S_{3}\right)+\mu^{2} d s^{2}\left(H^{4}\right), \\
\phi & =0 \\
G_{(4)} & =\frac{1}{2 g \mu^{2}} e^{1234}, \\
F^{A} & =-\frac{1}{g \mu^{2}}\left(e^{A} \wedge e^{4}+\frac{1}{2} \epsilon^{A B C} e^{B} \wedge e^{C}\right), \\
A^{A} & =\frac{r^{2}}{g\left(r^{2}-1\right)} \sigma^{A} .
\end{aligned}
$$

This solution admits precisely the two Killing spinors $\epsilon$ and $\epsilon_{K}=\left(g v+\Gamma^{-}\right) \epsilon$, and it may readily be verified that both of these spinors are null, as are their sum and difference. Uplifted to eleven dimensions, this solution is the $A d S$ fixed point of the near-horizon limit of an $M 5$ brane wrapped on a Cayley 4-cycle in a Spin(7) manifold [30].

Four Killing spinors Now suppose that in addition to the Killing spinors $\epsilon, \epsilon_{K}$, we demand the existence of the Killing spinor $T^{1} \epsilon$. This imposes $F^{2}=F^{3}=0$, also it implies the existence of the fourth Killing spinor $\epsilon_{K}^{\prime}=T^{1}\left(g v+\Gamma^{-}\right) \epsilon$. Since $A^{2}=A^{3}=0$, we now have

$$
\tilde{\nabla} J^{1}=0
$$

so the base must be chosen to be Einstein-Kähler, with $F^{1}=g^{-1} \mathfrak{R}$, where $\mathfrak{R}$ the Ricci form of the base. From the four form field equation we find $g=12 h$. The four spinors $\epsilon, T^{1} \epsilon, \epsilon_{K}, \epsilon_{K}^{\prime}$ are null. However we know from [19 that these solutions admit timelike spinors. It is easily checked that the four Killing spinors $\epsilon \pm \epsilon_{K}^{\prime}, T^{1} \epsilon \pm \epsilon_{K}$ are timelike. Uplifted to eleven dimensions, the metric is that of the $A d S$ fixed point of the near-horizon limit of an M5 wrapped on a Kähler 4-cycle in a Calabi-Yau 4-fold [30. 
Eight Killing spinors Finally, suppose that in addition to the four Killing spinors of the previous paragraph, we demand the existence of the Killing spinor $T^{2} \epsilon$. This imposes

$$
\begin{aligned}
F^{A} & =h=0, \\
G_{+-5} & =\omega_{-+5}=-\frac{g}{2}, \\
\nabla J^{A} & =0,
\end{aligned}
$$

and implies the existence of the additional Killing spinors $T^{3} \epsilon, T^{2}\left(g v+\Gamma^{-}\right) \epsilon, T^{3}(g v+$ $\left.\Gamma^{-}\right) \epsilon$. The three $J^{A}$ are required to be covariantly constant on the base, which must thus be taken to be Calabi-Yau. As in the previous example, these solutions admit both timelike and null Killing spinors. Lifted to ten dimensions, they are just the familiar $A d S_{3} \times S^{3} \times \mathcal{M}_{4}$ solutions of ten dimensional supergravity.

The $A d S$ examples we have given clearly illustrate the effect that the additional independent Killing spinors have on the torsion of the G-structure, which is $S U(2)$ in each case. The base is progressively restricted from Einstein to Einstein-Kähler to Calabi-Yau, together with an associated truncation of the Yang-Mills fields from $S U(2)$ to $U(1)$ to zero, as additional Killing spinors are incorporated.

\subsubsection{Generalisations: membranes with $A d S_{3}$ worldvolume}

Let us now consider generalising the known solutions given above, by allowing for a non-constant dilaton. In fact, the only additional truncation of the matter fields we will make, beyond that implied by the required supersymmetry, is to set $P_{1,2,3}^{(-)}=0$. Let us demand the existence of the second Killing spinor

$$
\epsilon_{K}=\left(g v+e^{-\phi} \Gamma^{-}\right) \epsilon .
$$

This implies that $\omega_{+5+}=\omega_{55+}=0$. Let us make the metric ansatz

$$
d s^{2}=L^{-1}(x, z) d u d v+C^{2}(x, z) d z^{2}+h_{m n}(x) d x^{M} d x^{N} .
$$

Now let us solve the constraints

$$
\omega_{-+i}=\omega_{5 i 5}=\partial_{i} \phi
$$

These read

$$
\frac{1}{2 L} \partial_{i} L=-\frac{1}{C} \partial_{i} C=\partial_{i} \phi
$$

which are solved by

$$
\begin{aligned}
L & =e^{2 \phi} \tilde{L}(z), \\
C & =e^{-\phi} \tilde{C}(z) .
\end{aligned}
$$


Next, the constraint

$$
\omega_{-+5}=-\frac{g}{2} e^{\phi},
$$

becomes

$$
\partial_{z} \tilde{L}=-g \tilde{C} \tilde{L}
$$

which as before is solved by

$$
\tilde{L}=(g z)^{-2}, \quad \tilde{C}=2 \tilde{L}^{1 / 2} .
$$

Given our metric ansatz, all the remaining constraints on the spin connection are satisfied, except for

$$
\omega_{i j k} J^{A i j}=2 g A_{i}^{A}-3 \partial_{j} \phi J_{i}^{A j} .
$$

Conformally rescaling the base according to

$$
h_{(4)}=e^{3 \phi} \tilde{h}_{(4)},
$$

we find

$$
\tilde{\omega}_{i j k} \tilde{J}^{A j k}=2 g A_{i}^{A},
$$

where $J_{i j}^{A}=e^{3 \phi} \tilde{J}_{i j}^{A}, \tilde{\omega}_{i j k}$ is the spin connection on the base with metric $\tilde{h}_{(4)}$, and for the remainder of this discussion all indices on "tilded" objects are raised with $\tilde{h}^{i j}$. The seven metric is thus

$$
d s^{2}=4\left(g e^{\phi}\right)^{-2}\left(z^{2}\left(-d t^{2}+d y^{2}\right)+\frac{d z^{2}}{z^{2}}\right)+e^{3 \phi} d \tilde{s}^{2},
$$

the warped product of $A d S_{3}$ with some four-manifold. Next, imposing the Bianchi identity for $F^{A}$ (which we recall in our conventions for the field strength is of the modified form $D\left(e^{\phi} F^{A}\right)=0$ ), we find that

$$
F_{i j}^{A}=\left(g e^{\phi}\right)^{-1} \tilde{\mathfrak{R}}_{i j}^{A},
$$

and as before that

$$
\tilde{R}=-16 h g,
$$

where the conformally rescaled metric $\tilde{h}$ is either Einstein, Kähler-Einstein or CalabiYau, depending on whether the Yang-Mills fields are $S U(2), U(1)$ or zero. It remains to impose the Bianchi identities and field equations for $G$. It is trivially verified that the four-form Bianchi identity, $d\left(e^{-2 \phi} G_{(4)}\right)=0$, is satisfied. Finally, the field equation for $G_{(4)}$ reduces to a single equation for $\phi$ :

$$
\frac{5}{2}\left(\tilde{\nabla}^{2} \phi+5 \tilde{h}^{i j} \partial_{i} \phi \partial_{j} \phi\right)-4 h g+h^{2} e^{-5 \phi}\left(32+\frac{16}{n}\right)=0,
$$

where $n=3$ for $S U(2)$ Yang-Mills fields and $n=1$ for $U(1)$. When the Yang-Mills fields vanish, we must take $h=0$. The examples in the previous subsection are 
clearly $\phi=0$ solutions of this equation. When $\phi$ is not constant, by making the definition

$$
\phi=\frac{1}{5} \log \left(f+\frac{4 h}{g}\left(2+\frac{1}{n}\right)\right)
$$

we find that $f$ obeys

$$
\nabla^{2} f-8 h g f=0
$$

As a first example of a more general solution of this form, take the conformally rescaled base to be $H^{4}$, with squared radius $\frac{3}{4 h g}$, and metric

$$
d \tilde{s}^{2}=\frac{3}{4 h g}\left(d r^{2}+\sinh ^{2} d d \Omega_{3}^{2}\right)
$$

We take $f=f(r)$, and thus obtain

$$
f^{\prime \prime}+3 \operatorname{coth} r f^{\prime}-6 f=0
$$

Making the change of variable $u=\tanh ^{2} r$, and defining $f=(1-u)^{\alpha} \psi, \alpha=$ $(3+\sqrt{33}) / 4$, converts this to hypergeometric form,

$$
u(1-u) \frac{d^{2} \psi}{d u^{2}}+[c-(1+a+b) u] \frac{d \psi}{d u}-a b \psi=0,
$$

with $a=(3+\sqrt{33}) / 4, b=(5+\sqrt{33}) / 4, c=2$. Depending on the solution we choose for $\psi$, we find that the metric is singular either at $r=0$ or $r=\infty$, or both. The $S U(2)$ Yang-Mills fields may be read off from (5.78). Imposing regularity at infinity, we choose $\psi=F\left(a, b ; 1+a+b-c ; 1-\tanh ^{2} r\right)$; we find that for large $r$, the solution asymptotes to our previous $A d S_{3} \times H^{4}$ example. To investigate the behaviour near $r=0$, we define a new radial coordinate $\rho=r^{2 / 5}$; the metric approaches that of a cone over $A d S_{3} \times S^{3}$,

$$
d s^{2}=d \rho^{2}+\rho^{2}\left(R_{1}^{2} d s^{2}\left(A d S_{3}\right)+R_{2}^{2} d s^{2}\left(S^{3}\right)\right),
$$

and the dilaton blows up. The full solution preserves two supersymmetries.

As a second example of a more general solution of this form, take the conformally rescaled base metric to be flat,

$$
d \widetilde{s}^{2}=d r^{2}+r^{2} d \Omega_{3}^{2}
$$

This choice implies that $F^{A}=h=0$. Let us set $f=f(r)$, so that up to an irrelevant multiplicative constant,

$$
f=1+\frac{a}{r^{2}}
$$

The seven dimensional metric is thus

$$
d s^{2}=4 g^{-2}\left(1+\frac{a}{r^{2}}\right)^{-2 / 5} d s^{2}\left(A d S_{3}\right)+\left(1+\frac{a}{r^{2}}\right)^{3 / 5} d s^{2}\left(\mathbb{R}^{4}\right) .
$$


Clearly, for large $r$ the metric becomes that of $A d S_{3} \times \mathbb{R}^{4}$. To investigate the behaviour near $r=0$, let us define $u=\frac{5}{2} a^{1 / 2} r^{2 / 5}$. Near $r=0$, the metric becomes

$$
d s^{2}=d u^{2}+u^{2}\left(\frac{16}{25 g^{2}} d s^{2}\left(A d S_{3}\right)+\frac{4}{25} d s^{2}\left(S^{3}\right)\right)
$$

which is again that of a cone over $A d S_{3} \times S^{3}$. Again, the dilaton blows up as we approach $r=0$. The full solution preserves eight supersymmetries.

\section{6. $\mathbb{R}^{5}$ structure}

Next consider an arbitrary linear combination of $(+,+)$ and $(+,-)$ spinors, which we denote by

$$
\left(\delta+i \delta^{A} T^{A}+\delta_{i} \Gamma^{i}\right) \epsilon
$$

and where at least one of the $\delta_{i} \neq 0$. It is convenient to introduce an orthonormal basis on the four dimensional base. Defining $H^{2}=\delta_{i} \delta^{i}$, we choose the basis

$$
e_{i}^{A}=H^{-1} J_{j i}^{A} \delta^{j}, A=1, . ., 3, \quad e_{i}^{4}=H^{-1} \delta_{i} .
$$

In this basis, we have $\epsilon^{1234}=1$. We will thus write $\epsilon^{A B C 4}=\epsilon^{A B C}$. To avoid potential confusion with this mixing of spacetime and Yang-Mills indices, we will always place Yang-Mills indices on the $J^{A}$ and the $F^{A}$ "up" and spacetime indices "down". However we will make no distinction between "up" and "down" indices on $\epsilon^{A B C}$. The components of the $J^{A}$ in this basis are

$$
J_{B 4}^{A}=-\delta^{A B}, \quad J_{B C}^{A}=-\epsilon^{A B C} .
$$

For computational convenience, we will restrict attention to Killing spinors which are not of the most general form compatible with an $\mathbb{R}^{5}$ structure, but which are rather of the form $\delta_{i} \Gamma^{i} \epsilon=H \Gamma^{4} \epsilon$. Evaluating

$$
\left[\Delta_{\lambda}, \delta_{i} \Gamma^{i}\right] \epsilon=0
$$

and employing the constraints of section three, we find the conditions

$$
\begin{aligned}
G_{-i j} & =G_{i j k}=0, \\
\epsilon^{A B C} F_{B C}^{A} & =20\left(m+2 h e^{-4 \phi}\right), \\
\epsilon^{A B C} F_{4 C}^{B} & =\epsilon^{A B C} G_{5 B C} .
\end{aligned}
$$

Next, using $\omega_{\mu}^{4}{ }_{\nu}=-\nabla_{\mu} e_{\nu}^{4}$, we deduce from commuting the - component of the Killing spinor equation that

$$
\partial_{-} H=0
$$


From the + component we find

$$
\begin{aligned}
\partial_{+} H & =h=0, \\
\omega_{+4 A} & =G_{+4 A}, \\
G_{45 A} & =\frac{1}{2} F_{B A}^{B} .
\end{aligned}
$$

The 5 component yields

$$
\begin{aligned}
\partial_{5} H & =0, \\
\omega_{54 A} & =G_{54 A},
\end{aligned}
$$

while the $B$ component gives

$$
\begin{aligned}
\partial_{B} H=F_{B C}^{A} & =g=0, \\
\omega_{B 4 A} & =\partial_{4} \phi g_{A B}, \\
\epsilon^{A C D} \omega_{B C D} & =\frac{3}{2} \partial_{4} \phi g_{A B}+\epsilon^{A B C} \partial_{C} \phi .
\end{aligned}
$$

Since the gauge coupling $g$ is required to be zero, an $\mathbb{R}^{5}$ structure of the form we have assumed is only admitted in the ungauged theory. The remaining constraints may be derived from the 4 component of the Killing spinor equation, and we find them to be of the form

$$
\begin{aligned}
H & =\text { const }, \\
\partial_{5} \phi & =0, \\
\epsilon^{A B C} F_{4 C}^{B} & =G_{5 i j}=0, \\
\omega_{4 A 4} & =\partial_{A} \phi .
\end{aligned}
$$

We have thus derived the complete set of additional constraints on a bosonic configuration for it to admit the second Killing spinor $\delta_{i} \Gamma^{i} \epsilon$. However, there are no solutions of this form in the gauged theory, since the coupling is zero. We have also verified that there are no vacuum solutions, or solutions with eight Killing spinors, with an $\mathbb{R}^{5}$ structure in the gauged theory. We will not consider this case any further.

\section{Identity Structure}

Making a more general ansatz for a second Killing spinor than that consistent with an $\left(S U(2) \ltimes \mathbb{R}^{5}\right) \times \mathbb{R}, S U(2)$ or $\mathbb{R}^{5}$ structure implies that the structure group is reduced to the identity. The generic such Killing spinor is parameterised by sixteen real functions. Computing the constraints associated with the existence of such a spinor is a lengthy computational exercise. To illustrate the case of an identity structure, we will instead restrict attention to Killing spinors of the simpler form

$$
\theta_{i} \Gamma^{-i} \epsilon
$$

that is, pure $(-,-)$ spinors, in the language of the introduction. 


\subsection{Constraints}

As in the previous section, we introduce an orthonormal basis on the base; as before, we define $H^{2}=\theta_{i} \theta^{i}$, and choose

$$
e_{i}^{A}=H^{-1} J_{j i}^{A} \theta^{j}, \quad e_{i}^{4}=H^{-1} \theta_{i}
$$

Commuting the dilatino variation, we may derive

$$
\begin{aligned}
\partial_{+} \phi=\partial_{i} \phi & =G_{+5 i}=G_{+i j}^{(-)}=0, \\
G_{+-5} & =\frac{1}{2} F_{4 A}^{A}, \\
G_{45 A} & =\frac{1}{2} \epsilon^{A B C} F_{4 C}^{B} .
\end{aligned}
$$

Now, from the + component of the Killing spinor equation, we get

$$
\partial_{+} H=\omega_{++\mu}=F_{4 A}^{A}=\epsilon^{A B C} F_{4 C}^{B}=\omega_{+i j}^{(-)}=0 .
$$

The 5 component gives

$$
\begin{aligned}
\omega_{5+5}=\omega_{5+i} & =\omega_{554}=\omega_{54 A}=0, \\
\partial_{5}(\log H+\phi) & =4 h e^{-4 \phi} .
\end{aligned}
$$

Next from the - component we find

$$
\begin{aligned}
\partial_{-} H=\omega_{-+i}=G_{-+i} & =G_{i j k}=G_{-i j}=0, \\
\epsilon^{A B C} G_{5 B C} & =F_{B A}^{B} .
\end{aligned}
$$

The $A$ component yields the constraints

$$
\begin{aligned}
\partial_{A} H=\omega_{A 54} & =\omega_{A B 4}=F_{4 B}^{A}=0, \\
\omega_{i j+} & =G_{i j+} .
\end{aligned}
$$

Finally the 4 component gives

$$
\partial_{4} H=\omega_{44 A}=0
$$

These, in addition to the constraints of section 2, are the full set of neccessary and sufficient conditions on the bosonic fields of the theory for the existence of a second Killing spinor of the form (7.1). We see that demanding the existence of such a Killing spinor, with its associated identity structure, implies a radical simplification of the general problem. In fact, we will make one further simplifying assumption. By inspection of the constraints on the spin connection, we see that $d e^{5}=0$ if and only if $\epsilon^{A B C} G_{5 B C}=F_{B A}^{B}=0$; we will assume that $G_{5 A B}=0$. Then since $d e^{5}=0$, we may always choose our local coordinates such that $e^{5}=d z$, that is, $C=1, \sigma=0$. 
Summary Given the assumption that $G_{5 A B}=0$, demanding the existence of the second Killing spinor (7.1) implies that we may choose coordinates such that $e^{5}=d z$, and that the matter fields and the function $H$ are of the following form:

$$
\begin{aligned}
\phi & =\phi(z), \\
H & =H(z), \\
G_{(3)} & =e^{+} \wedge Q^{(+)}, \\
F^{A} & =\frac{1}{2} F_{B C}^{A} e^{B} \wedge e^{C},
\end{aligned}
$$

where $Q^{(+)}$is a selfdual form on the base. There are the following constraints on $\phi$, $H$ and $F^{A}$ :

$$
\begin{aligned}
\partial_{z} \log \left(H e^{\phi}\right) & =4 h e^{-4 \phi}, \\
F_{A B}^{A} & =0, \\
\epsilon^{A B C} F_{B C}^{A} & =10\left(\partial_{z} \log H+2 m\right) .
\end{aligned}
$$

The only nonzero independent spin connection components are the following:

$$
\begin{aligned}
\omega_{-+5} & =-\partial_{z} \log H \\
\omega_{+i j} & =\omega_{+i j}^{(+)} \\
\omega_{i j+} & =Q_{i j} \\
\omega_{454} & =-\partial_{z} \log H \\
\omega_{A 5 B} & =\left(\frac{3}{2} \partial_{z} \log H+5 m\right) g_{A B}+\frac{1}{2} \epsilon^{C B D} F_{A D}^{C}-\frac{1}{4} \epsilon^{A D E} F_{D E}^{B}, \\
\omega_{i A B} & =-g \epsilon^{A B C} A_{i}^{C} .
\end{aligned}
$$

The degree of simplification in this case is quite remarkable.

\subsection{Examples}

Note that since $\omega_{(\mu \nu)+}=0, \frac{\partial}{\partial u}$ is Killing. We will for simplicity seek solutions with $\omega_{i j+}=G_{i j+}=0$. Then note that performing the rescalings

$$
e^{+}=H \tilde{e}^{+}, \quad e^{-}=H \tilde{e}^{-}, \quad e^{5}=H \tilde{e}^{5}, \quad e^{4}=H \tilde{e}^{4},
$$

the constraints on $\omega_{+-5}$ and $\omega_{454}$ imply that $d \tilde{e}^{-}=d \tilde{e}^{+}=d \tilde{e}^{4}=d \tilde{e}^{5}=0$; thus locally we may introduce coordinates such that

$$
\begin{aligned}
\tilde{e}^{+} & =d u, \\
\tilde{e}^{-} & =d v, \\
\tilde{e}^{4} & =d r, \\
\tilde{e}^{5} & =d z .
\end{aligned}
$$


Note that we have redefined the $z$ coordinate. Furthermore, since $u$ is Killing, the base is independent of $u$. We have thus solved all the constraints on the spin connection except

$$
\begin{aligned}
\omega_{A 5 B} & =\left(\frac{3}{2} H^{-1} \partial_{z} \log H+5 m\right) g_{A B}+\frac{1}{2} \epsilon^{C B D} F_{A D}^{C}-\frac{1}{4} \epsilon^{A D E} F_{D E}^{B}, \\
\omega_{i A B} & =-g \epsilon^{A B C} A_{i}^{C},
\end{aligned}
$$

which we have written in terms of our new $z$ coordinate.

The Yang-Mills Bianchi identities together with (7.15) then imply that, as in the case of the $S U(2)$ structure,

$$
e^{\phi} F_{i j}^{A}=\frac{1}{g} \mathfrak{R}_{i j}^{A},
$$

where the $\mathfrak{R}_{i j}^{A}$ are the curvatures of the right hand spin bundle of the base with metric

$$
d s^{4}=H^{2}(z) d r^{2}+\delta_{A B} e^{A} e^{B} .
$$

Recall that $F_{4 B}^{A}=0$, and in addition to (7.14), (7.15) we have the additional constraints, written in terms of our new $z$ coordinate:

$$
\begin{aligned}
\partial_{z} \log \left(H e^{\phi}\right) & =4 h H e^{-4 \phi}, \\
F_{A B}^{A} & =0, \\
\epsilon^{A B C} F_{B C}^{A} & =10\left(H^{-1} \partial_{z} \log H+2 m\right) .
\end{aligned}
$$

Let us now consider some explicit examples.

Two Killing spinors Recall that since the scalar curvature of the base is given by $R=J^{A i j} \Re_{i j}^{A}=g J^{A i j} e^{\phi} F_{i j}^{A}=-g e^{\phi} \epsilon^{A B C} F_{B C}^{A}$. Equation (7.20) implies that $R$ is a function only of $z$. Let us look for a solution with a base of the form

$$
H^{2}\left(d r^{2}+\rho^{2}(z) d s^{2}\left(S^{3}\right)\right),
$$

so that

$$
F_{B C}^{A}=-(H \rho)^{-2} e^{-\phi} \epsilon^{A B C}=\frac{5}{3}\left(H^{-1} \partial_{z} \log H+2 m\right) \epsilon^{A B C} .
$$

We will seek a solution with $h=0$, so that (7.18) implies that up to an irrelevant multiplicative constant, $H=e^{-\phi}$. Then writing $H=f(z) e^{g z / 5}$, the second equality of (7.22) becomes

$$
-\rho^{-2}=\frac{5}{3} \partial_{z} \log f
$$

Next, using the expression for the spin connection given in Appendix C, (7.14) is

$$
\partial_{z}(3 \log \rho+10 \log f)=0,
$$

and hence

$$
\rho^{3}=\beta f^{-10}
$$


for some constant $\beta$. Hence we find that

$$
f^{-20 / 3}=\frac{4}{\beta} z+\alpha,
$$

and so shifting $z$ to eliminate the constant $\alpha$, the metric and dilaton may be written as

$$
\begin{aligned}
d s^{2} & =\left(\frac{4 z}{\beta}\right)^{-3 / 10} e^{2 g z / 5}\left(-d t^{2}+d \mathbf{x}^{2}+d z^{2}+4 z d s^{2}\left(S^{3}\right)\right), \\
e^{\phi} & =\left(\frac{4 z}{\beta}\right)^{3 / 20} e^{-g z / 5} .
\end{aligned}
$$

This describes the near-horizon limit of a IIB fivebrane wrapped on an associative three-sphere [31].

Four Killing spinors Let now us seek $U(1)$ solutions; this is equivalent to demanding the existence of the Killing spinor $i T^{1} \epsilon$. Then we find that the $U(1)$ gauge field obeys $F_{1 A}^{1}=0$, and hence that $\omega_{A 51}=-H^{-1} \partial_{z} \log H \delta_{1 A}, \omega_{1 A 5}=\omega_{i A 1}=0$. Hence the configuration also admits the Killing spinor $H \Gamma^{-} \Gamma^{1}$. We rescale $e^{1}$, so that locally we can write the metric as

$$
d s_{7}^{2}=H^{2} d s^{2}\left(\mathbb{R}^{5}\right)+d s^{2}\left(\mathcal{M}_{2}\right) .
$$

Consider solutions with $\phi=0$. Then $H=-(4 h z)^{-1}, F_{23}=16 h-g$, and hence the scalar curvature of $\mathcal{M}_{2}$ is

$$
R=-\frac{g}{2}(16 h-g),
$$

and so $\mathcal{M}_{2}$ is either $\mathbb{R}^{2}, S^{2}$ or $H^{2}$, and is independent of the coordinates on the five othogonal directions. Hence we must impose $\omega_{A 5 B}=0, A, B=2,3$, and therefore $12 h=g$. Thus the solution is the $A d S_{5} \times H^{2}$ solution of Maldacena and Nunez [32], for which we have displayed the identity structure.

Eight Killing spinors Finally, let us look for solutions for which the Yang-Mills fields vanish. This is equivalent to demanding the existence of the eight Killing spinors $\epsilon, i T^{A} \epsilon, H \Gamma^{-} \Gamma^{i} \epsilon$. The vanishing of the Yang-Mills fields implies that $\omega_{A 5 B}=$ $-\partial_{z} \log H \delta_{A B}$. Then rescaling the $e^{A}$ by $H$, we see that locally the metric can be put in the form

$$
d s^{2}=H^{2} \eta_{\mu \nu} d x^{\mu} d x^{\nu} .
$$

Furthermore we must require

$$
\begin{aligned}
\partial_{z} \log H & =-2 m H, \\
\partial_{z} \log \left(H e^{\phi}\right) & =4 H h e^{-4 \phi} .
\end{aligned}
$$

Consider first the case of vanishing topological mass, $h=0$. Then up to irrelevant constants, $H=e^{-\phi}=\exp \frac{g z}{5}$. This solution is nothing but the reduction to seven dimensions of the linear dilaton solution in ten. 
When $h \neq 0$, let us look for a solution with $\phi=0$. We thus must have $16 h=g$, $H^{2}=(4 h z)^{-2}$. This is just the maximally supersymmetric $A d S_{7}$ solution of the theory.

\section{Conclusions and outlook}

In this work, a systematic formalism for performing complete G-structure classifications of supersymmetric bosonic configurations in supergravity theories has been presented, and illustrated in the context of $d=7, S U(2)$ gauged supergravity. The key notion for organising the classification is that of the common isotropy group of the additional Killing spinors. The formalism has been used to derive a set of constraints associated wth the existence of various additional Killing spinors, and these constraints have been exploited to derive numerous explicit solutions. The emphasis in this paper has been on illustrating the formalism, and no great effort has been made to derive new solutions; clearly, there is scope for a more careful analysis of the constraints in the future.

An important technical point that has been exploited throughout is that in performing G-structure classifications, it is unneccessary, and inefficient, to use spinor bilinears to derive the constraints; for more computational efficiency, it is much better to work directly with a specific spinor defined by a particular set of projections. The second key point that has been used is that given a single Killing spinor, any other spinor may be constructed from it by acting with a matrix $Q$ in the Clifford algebra. The problem of completely classifying all supersymmetric configurations in a given supergravity may then be restated as determining all possible sets of such matrices $Q$ which commute with the operators in the fermion supersymmetry transformations, when acting on the fiducial Killing spinor.

The chief advantage of the G-structure formalism used here over other classification techniques is that the constraints one derives for the existence of the desired supersymmetries take the form of explicit algebraic constraints on the spin connection and the matter fields, supplemented by first order differential conditions on the functions parameterising any additional Killing spinors. Presenting the neccessary and sufficient conditions for the existence of $N$ Killing spinors in this form naturally lends itself to exploiting the classification in the construction of explicit solutions, rendering G-structure classifications very useful for practical applications.

The only drawback of the formalism is, of course, the ammount of computational effort required to compute the constraints in a theory of the complexity of the one studied here. It is to be expected that simpler theories, such as those with eight supercharges, would be much more tractable from the point of view of perfoming a refined classification in full generality, using the techniques of this paper; this will be interesting to investigate. And it is of course possible that there is a yet more efficient way of computing the constraints. The approach employed here is "bottom 
up", in the sense that one first assumes the existence of a single Killing spinor, and then incorporates additional Killing spinors iteratively. For the analysis of more complicated supergravities, it would be very useful to have a complementary "top down" approach, so that one could start with maximal supersymmetry and weaken the constraints progressively, in a controlled fashion. This would be particularly useful for classifying configurations admitting more than one half supersymmetry, since in this case the structure group is neccessarily the identity, and this is the most complicated case to analyse using the iterative bottom up approach.

An obvious application of the formalism presented here is to the long-standing problem of completely classifying supersymmetric configurations in $d=11$. The possible structure groups in $d=11$ will coincide with the possible holonomy groups; these have been classified in [34], and there are eighteen distinct possibilites. A Gstructures analysis of the maximal structure groups $\left(S U(5)\right.$ and $\left.\left(\operatorname{Spin}(7) \ltimes \mathbb{R}^{8}\right) \times \mathbb{R}\right)$ has been given in [9], [10]. The first step in performing the complete classification will be to construct, from a fiducial Killing spinor, the spaces of spinors fixed by each of the other sixteen groups, as was done in the context of this paper in the introduction. Then it should certainly be practical to completely analyse the thirteen additional structure groups fixing at most eight Killing spinors; analysing the two groups $\left(S U(2)\right.$ and $\left.\mathbb{R}^{9}\right)$ fixing at most sixteen will require considerably more effort. Finally, completely classifying the most generic case of an identity structure (which fixes at most thirty-two Killing spinors) will, without further insight, require an immense ammount of calculation.

Nevertheless, a complete G-structure classification of all supersymmetric configurations in eleven dimensions (or in any other supergravity) will be of significant value. An in-depth analysis of the constraints derived from a standard classification of simple five dimensional supergravities has already yielded some very interesting solutions, such as supersymmetric $A d S_{5}$ black holes [35], [36], and supersymmetric black rings, [37-39. One would hope that a complete G-structure analysis in eleven dimensions would reveal a wealth of surprising new phenomena.

\section{Acknowledgements}

The author is supported by the National University of Ireland, EPSRC and a Freyer studentship. Stimulating discussions with Marco Cariglia during the early stages of this project are gratefully acknowledged.

\section{A. Conventions}

We work in almost plus signature, $\eta_{\mu \nu}=\operatorname{diag}(-,+, \ldots,+)$. Seven dimensional

spacetime indices are denoted by Greek letters $\mu, \nu, \ldots$ Orthonormal indices on 
the four dimensional base are denoted by lower case Roman letters, $i, j, \ldots$; spacetime indices on the base are denoted by upper case Roman letters $M, N, \ldots$ In an orthonormal frame the Dirac algebra is

$$
\left\{\Gamma_{\mu}, \Gamma_{\nu}\right\}=2 g_{\mu \nu} .
$$

This tells us that $\Gamma_{0}$ is antihermitian and the $\Gamma_{i}(i=1, \ldots, 6)$ are hermitian. Following the appendix to Chapter 1 in [33] we have that the charge conjugation matrix $C$ satisfies

$$
C^{T}=C, \quad C^{\dagger} C=\boldsymbol{I}, \quad \Gamma_{\mu}^{T}=-C \Gamma_{\mu} C^{-1} .
$$

We can therefore choose

$$
C=\boldsymbol{I} .
$$

This implies that $\Gamma_{0}$ is real and the $\Gamma_{i}$ are imaginary. We will choose a representation (there are two inequivalent ones) such that

$$
\Gamma_{0} \Gamma_{1} \Gamma_{2} \Gamma_{3} \Gamma_{4} \Gamma_{5} \Gamma_{6}=-\boldsymbol{I} .
$$

We also have the identity

$$
\Gamma_{\alpha_{1} \ldots \alpha_{n}}=\frac{(-)^{[n / 2]+1}}{(7-n) !} \epsilon_{\alpha_{1} \ldots \alpha_{n} \beta_{1} \ldots \beta_{7-n}} \Gamma^{\beta_{1} \ldots \beta_{7-n}} .
$$

We choose the orientation to be given by $\epsilon^{0123456}=+1$. Our null basis is defined by

$$
e^{ \pm}=\frac{1}{\sqrt{2}}\left(e^{0} \pm e^{6}\right)
$$

so that $\epsilon^{+-12345}=1$. The orientation on the base is chosen to be given by $\epsilon^{+-i j k l 5}=$ $\epsilon^{i j k l}$.

The Dirac conjugate $\bar{\epsilon}_{a}$ of an anticommuting spinor $\epsilon^{a}$ is defined as

$$
\bar{\epsilon}_{a}=\left(\epsilon^{a}\right)^{\dagger} \Gamma_{0},
$$

and we also define

$$
\bar{\epsilon}^{a}=\epsilon^{a b} \bar{\epsilon}_{b},
$$

where $\epsilon^{a b}$ is a constant antisymmetric matrix satisfying $\epsilon_{a b} \epsilon^{b c}=-\delta_{a}^{c}$ that is used to raise and lower spinor indices according to $\epsilon^{a} \equiv \epsilon^{a b} \epsilon_{b}$, and $\epsilon^{12}=1$. On the other hand the symplectic-Majorana conjugate $\epsilon^{C}$ of $\epsilon$ is defined to be

$$
\left(\epsilon^{C}\right)^{a}=\left(\epsilon^{T}\right)_{b} .
$$

Symplectic-Majorana spinors are those for which (A.8) is equal to (A.9), namely

$$
\left(\epsilon^{T}\right)^{a}=\bar{\epsilon}^{a} .
$$

Given four spinors $\epsilon_{1}, \ldots, \epsilon_{4}$, the Fierz identity is

$$
\overline{\epsilon_{1}} \epsilon_{2} \overline{\epsilon_{3}} \epsilon_{4}=\frac{1}{8}\left[\overline{\epsilon_{1}} \epsilon_{4} \overline{\epsilon_{3}} \epsilon_{2}+\overline{\epsilon_{1}} \Gamma_{\mu} \epsilon_{4} \overline{\epsilon_{3}} \Gamma^{\mu} \epsilon_{2}-\frac{1}{2} \overline{\epsilon_{1}} \Gamma_{\mu \nu} \epsilon_{4} \overline{\epsilon_{3}} \Gamma^{\mu \nu} \epsilon_{2}-\frac{1}{3 !} \bar{\epsilon}_{1} \Gamma_{\mu \nu \rho} \epsilon_{4} \overline{\epsilon_{3}} \Gamma^{\mu \nu \rho} \epsilon_{2}\right] .
$$




\section{B. Useful projections}

We give here the full set of projections satisfied by the fiducial basis spinor that we have employed in deriving the constraints.

$$
\begin{aligned}
\Gamma^{+} \epsilon & =0, \\
\Gamma^{+-} \epsilon & =-\epsilon, \\
\Gamma^{i j k l} \epsilon & =-\epsilon^{i j k l} \epsilon, \\
A_{i j}^{(-)} \Gamma^{i j} \epsilon & =0, \\
\Gamma^{i j k} \epsilon & =\epsilon^{i j k l} \Gamma_{l} \epsilon, \\
J_{i j}^{A} \Gamma^{i j} \epsilon^{a} & =8 i T^{A a} \epsilon^{b}, \\
T^{A a} \Gamma_{i} \epsilon^{b} & =-\frac{i}{2} J_{i j}^{A} \Gamma^{j} \epsilon^{a} .
\end{aligned}
$$

\section{Spin connection components}

The exterior derivatives of the basis one forms are given by

$$
\begin{aligned}
d e^{+} & =e^{+} \wedge\left(\mathcal{D} \log L+\partial_{u} \lambda\right)+e^{5} \wedge\left(-(L C)^{-1} \partial_{z} \lambda\right) \\
& +e^{+} \wedge e^{5} C^{-1} \partial_{z} \log L-L^{-1} \mathcal{D} \lambda, \\
d e^{-} & =e^{+} \wedge L\left(\frac{1}{2} \mathcal{D} \mathcal{F}+\frac{1}{2} \lambda \partial_{u} \mathcal{F}-\partial_{u} \nu+\sigma \partial_{u} \beta\right) \\
& +e^{5} \wedge C^{-1}\left(-\frac{1}{2} \lambda \partial_{z} \mathcal{F}+\partial_{z} \nu-\sigma \partial_{z} B-\mathcal{D} B\right) \\
& +e^{+} \wedge e^{5} L C^{-1}\left(\frac{1}{2} \partial_{z} \mathcal{F}-\partial_{u} B\right) \\
& +\mathcal{D} \nu-\frac{1}{2} \mathcal{D} \mathcal{F} \wedge \lambda-\mathcal{D} B \wedge \sigma \\
d e^{5} & =e^{+} \wedge\left(-C L \partial_{u} \sigma\right)+e^{5} \wedge\left(\partial_{z} \sigma-\mathcal{D} \log C\right) \\
& +e^{+} \wedge e^{5}\left(-L \partial_{u} \log C\right)+C \mathcal{D} \sigma, \\
d e^{i} & =\mathcal{D} e^{i}+e^{+} \wedge\left(-L \partial_{u} e^{i}\right)+e^{5} \wedge\left(C^{-1} \partial_{z} e^{i}\right) .
\end{aligned}
$$

Then the full set of nonzero spin connection components is

$$
\begin{aligned}
\omega_{-+5} & =\frac{1}{2 C} \partial_{z} \log L, \\
\omega_{-+i} & =\frac{1}{2}\left(\mathcal{D} \log L+\partial_{u} \lambda\right)_{i}, \\
\omega_{-5 i} & =\frac{1}{2 L C}\left(\partial_{z} \lambda\right)_{i}, \\
\omega_{-i j} & =\frac{1}{2 L} \mathcal{D} \lambda_{i j},
\end{aligned}
$$




$$
\begin{aligned}
& \omega_{++5}=L C^{-1}\left(\frac{1}{2} \partial_{z} \mathcal{F}-\partial_{u} B\right) \\
& \omega_{++i}=L\left(\frac{1}{2} \mathcal{D} \mathcal{F}+\frac{1}{2} \lambda \partial_{u} \mathcal{F}-p a_{u} \nu+\sigma \partial_{u} B\right)_{i}, \\
& \omega_{+-5}=\omega_{-+5} \text {, } \\
& \omega_{+-i}=\omega_{-+i} \text {, } \\
& \omega_{+5 i}=\frac{1}{2 C}\left(-\frac{1}{2} \lambda \partial_{z} \mathcal{F}+\partial_{z} \nu-\sigma \partial_{z} B-\mathcal{D} B+L C^{2} \partial_{u} \sigma\right)_{i}, \\
& \omega_{+i j}=L M_{[i j]}+\frac{1}{2}\left(\mathcal{D} \nu-\frac{1}{2} \mathcal{D} \mathcal{F} \wedge \lambda-\mathcal{D} B \wedge \sigma\right)_{i j}, \\
& \omega_{5+-}=\omega_{-+5}, \\
& \omega_{5+5}=L \partial_{u} \log C \\
& \omega_{5+i}=\omega_{+5 i}, \\
& \omega_{5-i}=\omega_{-5 i} \text {, } \\
& \omega_{55 i}=\left(\mathcal{D} \log C-\partial_{z} \sigma\right)_{i}, \\
& \omega_{5 i j}=-C^{-1} \Lambda_{[i j]}-\frac{1}{2} C \mathcal{D} \sigma_{i j}, \\
& \omega_{i+-}=\omega_{-+i}, \\
& \omega_{i+5}=-\omega_{+5 i}, \\
& \omega_{i+j}=L M_{(i j)}+\frac{1}{2}\left(\mathcal{D} \nu-\frac{1}{2} \mathcal{D} \mathcal{F} \wedge \lambda-\mathcal{D} B \wedge \sigma\right)_{i j}, \\
& \omega_{i-5}=-\omega_{-5 i} \text {, } \\
& \omega_{i-j}=\omega_{-i j} \text {, } \\
& \omega_{i 5 j}=-C^{-1} \Lambda_{(i j)}-\frac{1}{2} C \mathcal{D} \sigma_{i j}, \\
& \omega_{i j k}=\hat{\omega}_{i j k}+\sigma_{i} \Lambda_{[j k]}+\sigma_{[j} \Lambda_{k] i}+\sigma_{[j} \Lambda_{|i| k]} \\
& -\lambda_{i} M_{[j k]}-\lambda_{[j} M_{k] i}-\lambda_{[j} M_{|i| k},
\end{aligned}
$$

where $\hat{\omega}_{i j k}$ is the spin connection on the base.

\section{Integrability Conditions}

In 119 it was shown that we may obtain the following integrability condition from commuting the Killing spinor equation with $\delta \lambda$ :

$$
\begin{aligned}
\sqrt{5} \Gamma^{\mu}\left[\mathcal{D}_{\mu}, \Delta_{\lambda}\right] \epsilon^{a} & =\left(\frac{1}{2} P+\frac{1}{6} Q_{\mu \nu \sigma} \Gamma^{\mu \nu \sigma}+\frac{e^{2 \phi}}{96} d\left(e^{-2 \phi} G\right)_{\mu \nu \sigma \tau \rho} \Gamma^{\mu \nu \sigma \tau \rho}\right) \epsilon^{a} \\
& +\left(i R_{\mu}^{A} \Gamma^{\mu}+\frac{i e^{-\phi}}{6} D\left(e^{\phi} F^{A}\right)_{\mu \nu \sigma} \Gamma^{\mu \nu \sigma}\right) T_{b}^{A a} \epsilon^{b} \\
& +\sqrt{5}\left(\frac{1}{60} G_{\mu \nu \sigma \tau} \Gamma^{\mu \nu \sigma \tau} \delta_{b}^{a}+\frac{3 i}{5} F_{\mu \nu}^{A} \Gamma^{\mu \nu} T_{b}^{A a}+(8 h-g) \delta_{b}^{a}\right) \delta \lambda^{b}(\mathrm{D} .1)
\end{aligned}
$$


where $D$ denotes the gauge-covariant exterior derivative, $P, Q, R$ are defined by eqs.(2.9,2.10,2.11), and the dilaton, four form and two form field equations are respectively $P=0, Q=0, R^{A}=0$. It was also shown that the integrability condition for the Killing spinor equation is

$$
\begin{aligned}
\Gamma^{\nu}\left[\mathcal{D}_{\mu}, \mathcal{D}_{\nu}\right] \epsilon^{a} & =\left[-\frac{1}{2} E_{\mu \nu} \Gamma^{\nu}+e^{2 \phi} d\left(e^{-2 \phi} G\right)^{\nu \sigma \tau \rho \xi}\left(-\frac{1}{120} g_{\mu \nu} \Gamma_{\sigma \tau \rho \xi}\right.\right. \\
& \left.\left.+\frac{1}{200} \Gamma_{\mu \nu \sigma \tau \rho \xi}\right)+\frac{1}{10} Q^{\nu \sigma \tau}\left(\frac{1}{2} \Gamma_{\mu \nu \sigma \tau}-g_{\mu \nu} \Gamma_{\sigma \tau}\right)\right] \epsilon^{a} \\
& +\left[\frac{i e^{-\phi}}{5} D\left(e^{\phi} F^{A}\right)^{\nu \sigma \tau}\left(2 g_{\mu \nu} \Gamma_{\sigma \tau}+\frac{1}{6} \Gamma_{\mu \nu \sigma \tau}\right)\right. \\
& \left.-\frac{i}{5} R^{A \nu}\left(-4 g_{\mu \nu}+\Gamma_{\mu \nu}\right)\right] T^{A a}{ }_{b} \epsilon^{b} \\
& +\left[\partial_{\mu} \phi \delta_{b}^{a}-\frac{i}{25} F^{A \nu \sigma}\left(8 g_{\mu \nu} \Gamma_{\sigma}-\Gamma_{\mu \nu \sigma}\right) T_{b}^{A a}+\frac{2}{5} m^{\prime} \Gamma_{\mu} \delta_{b}^{a}\right. \\
& \left.+\frac{1}{25} G^{\nu \sigma \tau \rho}\left(-\frac{2}{3} g_{\mu \nu} \Gamma_{\sigma \tau \rho}+\frac{1}{4} \Gamma_{\mu \nu \sigma \tau \rho}\right) \delta_{b}^{a}\right] \sqrt{5} \delta \lambda^{b}=0
\end{aligned}
$$

where the Einstein equations are $E_{\mu \nu}=0$.

When a single null Killing spinor exists one may readily verify that is is sufficient to impose the Bianchi identities for the forms, the field equations for $G_{(4)}$, the + component of the Yang-Mills field equations and the ++ component of the Einstein equations. When additional Killing spinors are incorporated, more of the

field equations and Bianchi identities are identically satisfied; working out which ones may be done straightforwardly case by case.

\section{References}

[1] K. Tod, All metrics admitting supercovariantly constant spinors, Phys. Lett. B 121 241 (1983)

[2] J. Gauntlett, J. Gutowski, C. Hull, S. Pakis and H. Reall, All supersymmetric solutions of minimal supergravity in five dimensions, Class. Quant. Grav. 20 (2003) 4587

[3] J. Gauntlett and J. Gutowski, All supersymmetric solutions of minimal gauged supergravity in five dimensions, Phys. Rev. D68, 105009 (2003)

[4] D. Martelli and J. Sparks, G-Structures, fluxes and calibrations in M-theory, Phys. Rev. D68 085014 (2003)

[5] J. Gutowski, D. Martelli and H. Reall, All supersymmetric solutions of minimal supergravity in six dimensions, hep-th/0306235.

[6] M. Cariglia and O. A. P. Mac Conamhna, The general form of supersymmetric solutions of $N=(1,0) U(1)$ and $S U(2)$ gauged supergravities in six dimensions, Class.Quant.Grav 21 (2004) 3171, hep-th/0402055. 
[7] J. P. Gauntlett, D. Martelli, D. Waldram, Superstrings with Intrinsic Torsion, hep-th/0302158.

[8] M. Caldarelli and D. Klemm, All supersymmetric solutions of $N=2, D=4$ gauged supergravity, JHEP 0309019 (2003)

[9] J. Gauntlett and S. Pakis, The geometry of D = 11 Killing spinors, JHEP 0304039 (2003)

[10] J. Gauntlett, J. Gutowski and S. Pakis, The geometry of D $=11$ null Killing spinors, hep-th/0311112

[11] J. P. Gauntlett, D. Martelli, S. Pakis, and D. Waldram, G-Structures and Wrapped NS5-Branes, hep-th/0205050.

[12] J. P. Gauntlett, D. Martelli, J. Sparks and D. Waldram, Supersymmetric AdS solutions of M-theory, hep-th/0402153.

[13] S. L. Cacciatori, M. M. Caldarelli, D. Klemm and D. S. Mansi, More on BPS solutions of $N=2, D=4$ gauged supergravity, hep-th/0406238.

[14] A. Lukas and P.M. Saffin, M-theory compactification, fluxes and $A d S_{4}$, hep-th/0403235.

[15] G. Dall'Agata, On supersymmetric solutions of type IIB supergravity with general fluxes, hep-th/0403220.

[16] E. J. Hackett-Jones and D. J. Smith, Type IIB Killing spinors and calibrations, hep-th/0405098.

[17] A. R. Frey, Notes on SU(3) Structures in Type IIB Supergravity, hep-th/0404107.

[18] S. L. Cacciatori, M. M. Caldarelli, D. Klemm and D. S. Mansi, More on BPS solutions of $N=2, D=4$ gauged supergravity, hep-th/0406238.

[19] M. Cariglia and O. Mac Conamhna, Timelike Killing spinors in seven dimensions, hep-th/0407127.

[20] M. Grana, R. Minasian, M. Petrini and A. Tomasiello, Supersymmetric backgrounds from generalised Calabi-Yau manifolds, hep-th/0406137.

[21] M. J. Duff and J. T. Liu, Hidden spacetime symmetries and generalised holonomy in M-theory, Nucl.Phys. B674 (2003) 217-230, hep-th/0303140.

[22] C. Hull, Holonomy and symmetry in M-theory, hep-th/0305039.

[23] G. Papadopoulos and D. Tsimpis, The holonomy of the supercovariant connection and Killing spinors, JHEP 0307 (2003) 018, hep-th/0306117.

[24] P. K. Townsend and P. van Nieuwenhuizen, Gauged Seven-Dimensional Supergravity, Phys. Lett. B125 (1983) 41. 
[25] L. Mezincescu, P. K. Townsend and P. van Nieuwenhuizen, Stability Of Gauged D = 7 Supergravity And The Definition Of Masslessness In (Ads) In Seven-Dimensions, Phys. Lett. B143 (1984) 384.

[26] A. H. Chamseddine and W.A. Sabra, $D=7$ SU(2) gauged supergravity from $D=10$ supergravity, Phys.Lett.B476:415-419,2000, hep-th/9911180.

[27] H. Lü and C. N. Pope, Exact embedding of $N=1, D=7$ gauged supergravity in $D=11$, Phys.Lett. B467 (1999) 67-72, hep-th/9906168.

[28] P. K. Townsend, K. Pilch and P. Van Nieuwenhuizen, Self-duality in odd dimensions, Phys. Lett. B136 (1984) 38, Addendum-ibid B137 (1984) 443.

[29] R. L. Bryant, Pseudo-Riemannian metrics with parallel spinor fields and vanishing Ricci tensor, math.DG 0004073.

[30] J. P. Gauntlett, N. Kim and D. Waldram, M-Fivebranes Wrapped on Supersymmetric Cycles, Phys.Rev. D63 (2001) 126001, hep-th/0012195.

[31] B. S. Acharya, J. P. Gauntlett and N. Kim, Fivebranes wrapped on associative three-cycles, Phys.Rev. D63 (2001) 106003, hep-th/0011190.

[32] J. Maldacena and C. Nunez, Supergravity description of field theories on curved manufolds and a no go theorem, Int.J.Mod.Phys. A16 (2001) 822, hep-th/007018.

[33] A. Salam and E. Sezgin, "Supergravities In Diverse Dimensions. Vol. 1, 2."

[34] J. M. Figueroa-O'Farrill, Breaking the M-waves, Class.Quant.Grav. 17 (2000) 2925, hep-th/9904124.

[35] J. B. Gutowski and H. S. Reall, Supersymmetric AdS5 black holes, JHEP 0402 (2004) 006, hep-th/0401042.

[36] J. B. Gutowski and H. S. Reall, General supersymmetric AdS5 black holes, JHEP 0404 (2004) 048, hep-th/0401129.

[37] H. Elvang, R. Emparan, D. Mateos and H. S. Reall, A supersymmetric black ring, hep-th/0407065.

[38] J. P. Gauntlett and J. B. Gutowski, Concentric black rings, hep-th/0408010.

[39] J. P. Gauntlett and J. B. Gutowski, General Concentric Black Rings, hep-th/0408122. 